\title{
Evaluación Integrada de pensamiento crítico y conciencia ciudadana como competencias ATC21s en Costa Rica y en Japón
}

Integrated Assessment of critical thinking and citizen self-awareness as ATC21s skills in Costa Rica and Japan

\author{
Volumen 22, Número 1 \\ Enero - Abril \\ pp. 1-39
}

\section{Aaron Elí Mena Araya}

\section{Citar este documento según modelo APA}

Mena Araya, Aaron Elí. (2022). Evaluación integrada de pensamiento crítico y conciencia ciudadana como competencias ATC21s en Costa Rica y en Japón. Revista Actualidades Investigativas en Educación, 22(1), 1-39. Doi. https://doi.org/10.15517/aie.v22i1.49067 


\title{
Evaluación Integrada de pensamiento crítico y conciencia ciudadana como competencias ATC21s en Costa Rica y en Japón
} Integrated Assessment of critical thinking and citizen self-awareness as ATC21s skills in Costa Rica and Japan

\author{
Aaron Elí Mena Araya ${ }^{1}$
}

Resumen: El aprendizaje basado en competencias en educación primaria requiere la formación integrada de múltiples competencias. Debido a su complejidad inherente, su evaluación supone retos importantes. Este artículo presenta los resultados de experiencias de formación y evaluación realizadas en escuelas de Costa Rica y de Japón durante el año escolar del 2019, dirigidas a desarrollar competencias ATC21s (Evaluación y Enseñanza de las Competencias del Siglo XXI) relacionadas con conciencia ciudadana, pensamiento crítico y literacidad en TIC. En cada país, un grupo experimental (26 estudiantes en Costa Rica y 27 en Japón) participó en dos unidades de aprendizaje, que incorporaron cortos animados, organizadores gráficos e historietas para abordar problemas comunitarios. Los resultados de ambas unidades fueron evaluados mediante el análisis de historietas $y$ cuestionarios. Los grupos de control (25 estudiantes en Costa Rica y 27 en Japón) participaron solo en los cuestionarios, los cuales se centran en los conceptos de comunidad y participación ciudadana. El análisis de historietas se enfocó en la conciencia ciudadana y en las habilidades de pensamiento crítico de las personas participantes, y el análisis de cuestionarios se centró en nociones vinculadas con comunidad y participación ciudadana. En Costa Rica, el análisis de las historietas mostró incrementos significativos en los puntajes de pensamiento crítico (PPC) y conciencia ciudadana (PCC) entre las dos unidades. En las dos unidades, el estudiantado japonés mostró un mejor desempeño en PPC y PCC que sus pares costarricenses. En ambos países se encontraron correlaciones significativas entre PPC y PCC. En los grupos experimentales y de control de ambos países, el análisis de los cuestionarios no mostró resultados concluyentes. Estos resultados muestran que la narrativa es más adecuada que la descripción y la exposición para que las personas estudiantes expresen actitudes activas y críticas relacionadas con ciudadanía y democracia.

Palabras clave: Aprendizaje basado en competencias, pensamiento crítico, ciudadanía, evaluación.

Abstract: Competency-based learning in elementary education requires the integrated instruction of multiple competencies. Because of its inherent complexity, the assessment of such instruction poses significant challenges. This study presents the results of instructional and assessment experiences implemented in elementary schools of Costa Rica and Japan during the 2019 school year. These activities aimed at developing ATC21s competencies related to local and global citizenship, critical thinking, and ICT literacy. In each country, an experimental group (26 students in Costa Rica and 27 in Japan) participated in two learning units that incorporated animated shorts, graphic organizers, and comics to address community issues. The results from both units are assessed through the analysis of comics and questionnaires. Control groups (25 students in Costa Rica and 27 in Japan) participated only in the questionnaires, which focused on concepts related to community and citizen participation. The comics' analysis focused on the participants' citizens self-awareness and critical thinking skills. The questionnaires' analysis dealt with attitudes related to community and citizen participation. In Costa Rica, the analysis of the comics showed significant increases in critical thinking (PPC) and citizen self-awareness (PCC) scores in the second unit. In both units, Japanese students showed better performance in PPC and PCC than their Costa Rican peers. In both countries, correlations were found between PPC and PCC. The analysis of the questionnaires did not show conclusive results in either country. These results suggest that narrative is more suitable than description and exposition for students to express active and critical attitudes related to citizenship and democracy.

Keywords: Competency-based learning, critical thinking, citizenship, assessment.

\footnotetext{
1 Profesor Asociado de la Universidad de Costa Rica, Escuela de Ciencias de la Comunicación Colectiva, San José, Costa Rica. Doctorando en Educación Escolar, Universidad de Tsukuba, Tsukuba, Japón. Orcid: https://orcid.org/0000-0002-2577$\underline{2221}$
}

Dirección electrónica: aaron.mena.araya@gmail.com

Artículo recibido: 30 de junio, 2021

Enviado a corrección: 01 de octubre, 2021 


\section{Introducción}

El modelo para la Evaluación y Enseñanza de las Competencias del Siglo XXI (en adelante, ATC21s) busca definir las competencias relevantes para la vida ciudadana en el siglo XXI. Fue desarrollado principalmente en la Universidad de Melbourne, con la colaboración de más de 60 instituciones de investigación (Cisco, 2010). En 2010, Australia, Finlandia, Portugal, Singapur y el Reino Unido implementaron los primeros proyectos piloto en sus escuelas. En 2012, se publicó un documento que detalla las competencias incluidas en el modelo y propone métodos de evaluación afines. Ese mismo año, Costa Rica adoptó el modelo como referente para el diseño instruccional y curricular en educación primaria y secundaria.

El modelo ATC21s plantea "formas de pensar", como la creatividad, el pensamiento crítico, la resolución de problemas y la toma de decisiones; "formas de trabajar", como la comunicación y la colaboración; y "herramientas para trabajar", que incluyen varias formas de literacidad mediática. También promueve competencias relacionadas con las "formas de vivir en el mundo", relacionadas con ciudadanía global y local, vida y carrera, y responsabilidad personal y social. El modelo también detalla indicadores para conocimientos, habilidades, actitudes, valores y éticas incluidos en cada competencia (Griffin et al., 2012).

La instrucción de las competencias ATC21s tiene dos enfoques fundamentales (Kamiya, 2014). El primero enfatiza los objetivos y evaluaciones de aprendizaje. El segundo se enfoca en la aparición emergente de nuevas competencias, y busca proporcionar entornos de aprendizaje en los que las personas estudiantes puedan establecer sus propios objetivos de aprendizaje y obtener los conocimientos que necesitan para afrontar los retos del siglo XXI. De manera general, la instrucción y la evaluación de las competencias ATC21s presuponen una progresión de aprendizaje (Wilson et al., 2016) en la que los individuos expresan niveles crecientes de sofisticación en diversas competencias a medida que se desarrollan cognitivamente, y participan en múltiples actividades de aprendizaje adecuadamente planificadas. En otras palabras, no se espera que las competencias del siglo XXI se adquieran a través de actividades de aprendizaje aisladas, sino que se cultiven a lo largo de un periodo extendido, a través de tareas cada vez más complejas.

La evaluación en el marco del ATC21s también se aborda desde distintas perspectivas. Los enfoques iniciales propusieron un análisis cualitativo de datos, centrado en los productos creados por las personas estudiantes. Uno de estos enfoques analizó el dominio de diversas tareas y el nivel de colaboración expresados en los cuadernos de estudiantes de distintos rangos de edad (Short, 2012). Otras experiencias adoptaron esta metodología para evaluar 
cómo las personas estudiantes colaboran al resolver asignaciones de matemáticas y ciencias en línea (Scalise, 2016). En concreto, se utilizaron los cuadernos de las personas estudiantes para evaluar el nivel de dominio en diversas tareas expresado por estudiantes de Estados Unidos de América, Singapur y Australia, al tiempo que se buscaba identificar patrones y tendencias en las habilidades de colaboración de las personas estudiantes.

En algunos países, las competencias ATC21s se han incorporado a proyectos preexistentes de evaluación longitudinal. En Australia, las competencias generales relacionadas con la literacidad en tecnologías de la información y la comunicación (en adelante, literacidad en TIC) se evalúan mediante tareas organizadas en diferentes niveles de complejidad y cuestionarios centrados en la vida escolar y familiar (Oyanagi, 2016). Las evaluaciones se realizan en línea y están dirigidas a estudiantes de sexto y décimo año, ya que buscan determinar el nivel de desarrollo de las personas estudiantes a lo largo de los años. Los resultados de estas evaluaciones se utilizan para descubrir las áreas de instrucción que necesitan ser mejoradas. Experiencias similares enfatizan la evaluación colaborativa y las redes sociales interactivas digitales en secundaria (Wilson et al., 2017).

Otros enfoques de evaluación incorporan el uso de videojuegos en línea. Estos incluyen análisis cualitativos del comportamiento de las personas estudiantes y extraen conclusiones sobre sus competencias en colaboración y resolución de problemas (Care et al., 2016). Los videojuegos reflejan los problemas del mundo real, lo que permite a las personas usuarias ejercitar su pensamiento de orden superior mientras exploran múltiples soluciones a problemas de naturaleza ambigua (Anwal y Griffin, 2018). Enfoques adicionales se basan en la aplicación de encuestas dirigidas a estudiantes de educación secundaria. En una de estas experiencias, las personas estudiantes describieron su propio nivel de dominio sobre múltiples competencias incluidas en el modelo ATC21s (Koike, 2019). Enfoques recientes incorporan modelos de instrucción dirigidos a apoyar la indagación científica, al tiempo que utilizan encuestas para determinar las actitudes de las personas estudiantes hacia el proceso de aprendizaje y sus capacidades para la resolución de problemas (Lam et al., 2020).

Cabe señalar que muchas experiencias de evaluación de aprendizajes dentro del modelo ATC21s se centran en actitudes, conocimientos y habilidades relacionadas con la colaboración, la resolución de problemas y la literacidad en TIC. Asimismo, la mayoría de las metodologías se basan en el análisis de la interacción entre las personas estudiantes durante la resolución de tareas, o buscan que las personas estudiantes reporten sobre sus propias habilidades a través de cuestionarios. De este modo, se puede afirmar que existe una ruta 
abierta para el desarrollo de nuevos métodos de evaluación enfocados en distintos acercamientos al modelo ATC21s. Dichos acercamientos pueden incluir la introducción de recursos que permitan a las personas estudiantes plasmar sus ideas a través de la narrativa, y procesos de aprendizaje que permitan a las personas estudiantes expresar individualmente sus habilidades de resolución de problemas.

Esta investigación tiene dos objetivos. Primero, busca facilitar el aprendizaje de las competencias ATC21s en escuelas primarias costarricenses y japonesa mediante el desarrollo de los conocimientos procedimentales y actitudes que las personas estudiantes necesitan para aplicar su pensamiento crítico en confluencia con su conciencia ciudadana. Adicionalmente, propone y aplica métodos novedosos para la evaluación integrada de las competencias ATC21s relacionadas con pensamiento crítico y conciencia ciudadana, que incorporan el uso de la narrativa y la visualización de pensamiento.

Cabe señalar que, aunque estos dos objetivos presentan diferencias en su naturaleza y en sus alcances, persiguen hallazgos complementarios. El primero tiene un carácter situado y finito, reflejado en aprendizajes concretos, y circunscrito en las prácticas y condiciones específicas de las escuelas y comunidades participantes. Por su parte, el segundo objetivo tiene un carácter abierto y extensivo, ya que utiliza los resultados de la evaluación de los aprendizajes no solamente para discutir particularidades de la educación y cultura de Costa Rica y Japón, sino también para discutir la posibilidad de extrapolar dichos resultados a otras realidades institucionales y culturales. En este sentido, mediante la consecución del primer objetivo se busca generar datos y reflexiones que permitan sustentar el cumplimiento del segundo objetivo.

Es importante mencionar que, debido la complejidad del aprendizaje basado en competencias, es necesario definir metas concretas para los procesos de instrucción y evaluación. Considerando este punto, esta investigación propone dos unidades de aprendizaje centradas en actitudes y formas de conocimiento específicas, regularmente aplicadas en la resolución de problemas en contextos de comunidad.

\section{Referente teórico}

\subsection{El aporte del modelo ATC21s a la educación ciudadana en Costa Rica y en Japón}

Los modelos basados en competencias presuponen la instrucción y evaluación simultánea de múltiples competencias. Estas formas de instrucción y evaluación implican la 
identificación de temas y contenidos adecuados para el aprendizaje de competencias transversales y la definición de resultados de aprendizaje específicos. También, requieren la introducción de medios educativos personalizados que aborden situaciones de la vida real, cercanas a las personas estudiantes. La complejidad de estas tareas plantea importantes retos metodológicos y conceptuales para las personas docentes, y dificulta el diseño de experiencias de aprendizaje que puedan adaptarse y reproducirse en diferentes contextos geográficos y culturales.

Los modelos basados en competencias pueden desempeñar un papel fundamental en la formación de ciudadanos activos, dispuestos y capaces de resolver problemas locales y globales. El modelo ATC21s incluye la ciudadanía, local y global, como una de sus competencias. El desarrollo de este tipo de ciudadanía está directamente apoyado por otras competencias incluidas en el modelo, como "Pensamiento crítico, resolución de problemas, toma de decisiones" y "Literacidad en TIC". La presente investigación propone que la instrucción y evaluación del pensamiento crítico y la conciencia ciudadana pueden abordarse eficazmente mediante actividades de aprendizaje que impliquen la activación de habilidades específicas relacionadas con estas competencias complementarias.

Figura 1. Integración de competencias ATC21s en la educación ciudadana

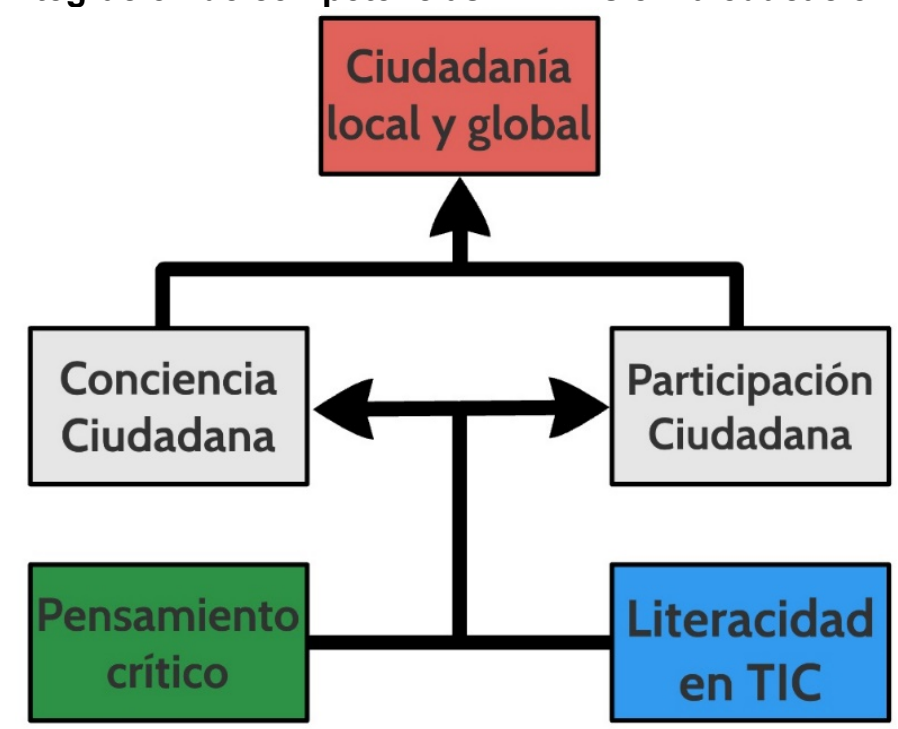

Fuente: Elaboración propia (2021).

La activación de la ciudadanía local y global requiere la adquisición de conciencia ciudadana, ya que esta se convierte en la motivación para interactuar responsablemente con la sociedad. También exige una participación ciudadana activa, que se relaciona con el trabajo 
en comunidad, el activismo político, la participación en procesos electorales y la creación de política pública. Además, la conciencia ciudadana y la participación ciudadana implican la activación de conocimientos, habilidades y actitudes relacionadas con el pensamiento crítico, la resolución de problemas, la toma de decisiones y la literacidad en TIC. En resumen, el pensamiento crítico y la literacidad en TIC promueven la conciencia ciudadana y la participación ciudadana, las cuales, al mismo tiempo, permiten el fortalecimiento de nociones de ciudadanía local y global (Figura 1).

Teniendo en consideración la adaptabilidad de los marcos basados en competencias, esta investigación integra múltiples elementos del marco ATC21s para abordar la instrucción y evaluación del pensamiento crítico y la consciencia ciudadana en escuelas primarias de Costa Rica y Japón. Este abordaje tiene dos méritos fundamentales. Primero, la integración de competencias ATC21s permite contrastar resultados de instrucción y evaluación en Costa Rica y Japón, dos países con sistemas educativos profundamente distintos.

La educación escolar costarricense tiene tres niveles: educación preescolar (dos años), educación primaria (seis años) y educación secundaria (cinco años). En el caso de la educación primaria, se ofrecen cuatro asignaturas regulares (Español, Matemáticas, Estudios Sociales y Ciencias) impartidas por uno o dos docentes de grado, y siete asignaturas especiales (Inglés, Música, Educación para el Hogar, Educación Religiosa, Artes plásticas, Educación Física e Informática), la mayoría de ellas impartidas por docentes especializados. Sin embargo, muchas escuelas primarias carecen, parcial o totalmente, de las asignaturas especiales, debido a condiciones presupuestarias y sociodemográficas. Cuando se compara con otros países de América Latina, Costa Rica muestra un rendimiento relativamente alto. Actualmente ocupa el puesto 62 de 189 países y regiones en el índice de educación (United Nations Development Programme, 2020). También tiene una tasa de alfabetización del 97,9\%, ligeramente por detrás de Cuba $(99,8 \%)$, Argentina $(99,7 \%)$ y Uruguay $(98,7 \%)$. En cuanto al acceso a Internet en las escuelas primarias (59\%) y secundarias (61\%), Costa Rica ocupa una posición de liderazgo, sólo superada por Chile y Uruguay.

Los principales problemas académicos de la educación costarricense incluyen altas tasas de repetición y de abandono escolar, tanto en primaria como en secundaria. Estos problemas suelen estar relacionados con bajos niveles de ingreso y bajos niveles educativos en los hogares de las personas estudiantes, y con la baja proporción de docentes por estudiante en las comunidades rurales (Loría y Martínez, 2018). En la educación secundaria, el bajo rendimiento de Costa Rica en las pruebas PISA llama la atención sobre problemáticas 
adicionales, relacionadas con limitaciones generalizadas en la enseñanza basada en competencias. En 2018, de 78 países participantes, Costa Rica obtuvo el puesto 49 en Lectura, el 60 en Ciencias y el 63 en Matemáticas (Schleicher, 2018).

Entre los problemas específicos relacionados con la instrucción de competencias ATC21s en la educación costarricense sobresalen la falta de sistemas para mapear el progreso de los estudiantes, la ausencia generalizada de herramientas de evaluación formativa disponibles para las personas docentes, el énfasis en las calificaciones por encima de los resultados de aprendizaje y la incomprensión general de la naturaleza longitudinal del aprendizaje basado en competencias (Portillo, 2017). Cabe resaltar que la evaluación de múltiples competencias en el contexto de cualquier asignatura escolar implica, como mínimo, la selección de ítems adecuados del currículo de la asignatura, la identificación y combinación de competencias que puedan relacionarse con los ítems seleccionados de forma adecuada, la selección de indicadores de aprendizaje adecuados para las competencias implicadas y la operacionalización de estos indicadores en actividades de evaluación específicas. En Costa Rica, las personas educadoras rara vez tienen la oportunidad de dedicar a este proceso la cantidad significativa de tiempo que requiere, o tienen acceso regular a medios educativos que puedan para apoyar la evaluación de competencias.

En muchas de estas áreas, Japón muestra diferencias significativas con Costa Rica. Con una tasa de alfabetización cercana al 100\% (Roser y Ortiz-Ospina, 2018), Japón ocupa el puesto 19 en el índice de educación. En las pruebas PISA de 2018, las personas estudiantes japonesas obtuvieron el puesto 13 en Lectura, el 4 en Ciencias y el 3 en Matemáticas, recibiendo la tercera puntuación combinada más alta a nivel mundial. En Japón, el abandono escolar es bajo, y la repetición en la educación primaria y secundaria es exigua (Ikeda y García, 2014). Los docentes de grado de primaria se encargan de todas las asignaturas regulares (Lengua Japonesa, Aritmética y Matemáticas, Estudios Sociales y Ciencias) y de la mayoría de las asignaturas especiales (Estudios del Entorno de la Vida, Música, Artes y Artesanías, Educación para el Hogar, Educación Física, Educación Moral, Periodo de Estudios Integrados y Educación Informática entre otras) (Murata y Yamaguchi, 2010).

La asignatura de Periodo de Estudios Integrados es uno de los elementos distintivos del sistema escolar japonés, y brinda un espacio ideal para el aprendizaje transversal de competencias. En esta asignatura, los niños y niñas participan en actividades de aprendizaje y proyectos en los que pueden aplicar los conocimientos obtenidos en otras asignaturas para resolver problemas complejos (Takamiya, 2015). Asimismo, el currículo de Estudios Sociales 
para primaria define una serie de competencias cívicas vinculadas a la comprensión de los propios derechos y deberes, y en el respeto a los otros miembros de la comunidad (Karaki, 2016). Estas competencias suelen desarrollarse mediante proyectos de aprendizaje de servicio, en los cuales el estudiantado busca dar soluciones a problemas de su comunidad mientras interactúan con múltiples instituciones y actores sociales.

Las amplias disparidades entre Costa Rica y Japón proporcionan una oportunidad valiosa para explorar cómo métodos similares de instrucción y evaluación basados en competencias pueden interactuar con diferentes condiciones escolares. Más específicamente, mediante la implementación de unidades de homólogas en Costa Rica y Japón, se busca demostrar la adaptabilidad de nuevos métodos de instrucción y evaluación, y abrir una discusión sobre las posibilidades de implementar métodos similares en escuelas de todo el mundo.

El segundo mérito de la integración de competencias ATC21s se relaciona con la atención que esta investigación pone sobre objetivos y contenidos reconocidos por los currículos escolares de diversos países, incluyendo a Costa Rica y Japón. A pesar de la ubicuidad de la educación cívica y ciudadana en los sistemas escolares de todo el mundo, muchos estudiantes carecen de los conocimientos, las habilidades y la motivación para participar en procesos electorales, en la construcción de la comunidad y en otras formas de compromiso político (Schulz, 2012). Incluso democracias liberales con importantes diferencias culturales y económicas, como Costa Rica y Japón, parecen compartir grandes dificultades para empoderar a sus ciudadanos (Fundación Japón, 2019; Programa de Naciones Unidades para el Desarrollo, 2013). La reticencia de las generaciones más jóvenes a involucrarse en temas políticos y sociales sugiere la necesidad de repensar la forma en que se aborda la ciudadanía en la educación primaria y secundaria. Esta investigación propone que la instrucción y evaluación integrada de competencias relacionadas con la formación ciudadana, el pensamiento crítico y la alfabetización en TIC permiten responder a esta necesidad.

\subsection{Integración de competencias ATC21s en la instrucción y evaluación del pensamiento crítico y la conciencia ciudadana}

En esta subsección se describen consideraciones teóricas sobre la integración las competencias de "ciudadanía global y local", "literacidad en TIC", y "pensamiento crítico, resolución de problemas y toma de decisiones" en la instrucción y evaluación del pensamiento 
crítico y la conciencia ciudadana. Asimismo, se detalla la manera en que estas competencias se complementan mutuamente.

\subsubsection{Ciudadanía global y local}

El modelo ATC21s define la comprensión de los conceptos y mecanismos relacionados con la ciudadanía y la democracia, la capacidad de resolver problemas locales y globales, y la participación en actividades comunitarias y procesos electorales como los principales conocimientos y habilidades relacionados con la competencia de ciudadanía local y global. El diseño de actividades de aprendizaje que aborden estos conocimientos y destrezas requiere, en primer lugar, claridad en torno a la definición de ciudadanía. Rubin y Giarelli (2015) reconocen dos conceptos contradictorios de "buena ciudadanía" dentro de la educación ciudadana. Mientras que los conceptos más convencionales hacen hincapié en la adquisición de conocimientos sobre instituciones políticas y sobre la importancia de respetar las leyes y otras normas, los conceptos más recientes promueven los movimientos sociales como mecanismos para mejorar las condiciones de las comunidades y el medio ambiente.

Giroux (1989), por su parte, considera a la ciudadanía como un proceso dialógico. Agrega que las escuelas deben funcionar como esferas públicas democráticas, donde las personas estudiantes puedan aprender las habilidades que necesitan para construir formas más emancipadoras de vida comunitaria. Es posible afirmar que el diseño de actividades de aprendizaje destinadas a apoyar la enseñanza de la ciudadanía global y local, debe abordarse desde lo que Giroux describe como pedagogía crítica.

Los puntos de vista de Giroux sobre la educación ciudadana resuenan con la perspectiva de Freire (2017) sobre los objetivos y mecanismos de la educación. Freire sostiene que la práctica pedagógica debe reconocer la omnipresencia de relaciones desiguales entre clases sociales y naciones, y trabajar activamente para lograr sociedades más inclusivas y democráticas. Esta investigación propone que el diseño de las actividades de aprendizaje destinadas a apoyar la instrucción de la ciudadanía global y local debe incorporar los principios propuestos por Giroux y Freire. Así, esta instrucción debe ir más allá de las nociones convencionales de educación ciudadana, y brindar a las personas estudiantes oportunidades para reflexionar sobre sus propias experiencias personales y proponer soluciones a los problemas que afectan a sus comunidades.

Es importante mencionar que las discusiones sobre conflictos de escuela y comunidad también pueden contribuir a la consolidación de prácticas de educación inclusiva. Estas 
prácticas buscan atender las necesidades de los estudiantes más vulnerables, valorando las experiencias personales de cada niño y niña, y reconociéndoles como participantes activos en la comunidad escolar (Korsgaard et al., 2020). De este modo, los niños y niñas pueden desarrollar sus competencias ciudadanas en un ambiente inclusivo, que favorece no solamente el aprendizaje de contenidos curriculares, sino también el fortalecimiento de vínculos interpersonales. Esta investigación propone que la formación de competencias relacionadas con ciudadanía debe facilitar también la inclusión educativa, mediante la realización de actividades de aprendizaje y evaluación, en las que cada estudiante pueda expresar su propia voz en discusiones sobre temas de interés compartido.

\subsubsection{Literacidad en $T I C$}

La literacidad en TIC implica la creación de productos mediáticos y el uso de herramientas digitales adecuadas para interpretar y expresar ideas en contextos diversos y multiculturales. También incluye la aplicación de conocimientos sobre las características de los distintos medios de comunicación. Dependiendo de cómo organizan y transmiten la información, los productos mediáticos pueden ser comprendidos como instancias del discurso descriptivo, narrativo y expositivo (Brewer, 1995). Mientras que la narrativa suele presentar secuencias de acontecimientos donde se expresan intenciones y acciones de personajes, la descripción transmite información visual y espacial, y la exposición organiza información de forma abstracta y lógica. La presente investigación sugiere que la narrativa es la forma de discurso más adecuada para ayudar a las personas estudiantes a pensar sobre cuestiones de ciudadanía local y global.

Las historias, como expresión fundamental del discurso narrativo, tienen efectos positivos en la motivación para el aprendizaje (Sunayama et al., 2017). También funcionan como puentes entre lo abstracto y lo concreto (Egan, 1989), y entre la fantasía y la realidad (Tomita, 2002). Las historias permiten a las personas estudiantes aplicar conceptos abstractos, como valores morales y sentidos de linealidad y conclusión, a situaciones ficticias que emulan experiencias de la vida real.

Las actividades narrativas en las que las personas estudiantes emplean medios digitales para presentar sus puntos de vista también pueden contribuir al desarrollo de habilidades de resolución de problemas y de pensamiento crítico (Sarica y Usluel, 2016). Además, al asumir el papel de creadores de medios, las personas estudiantes pueden dominar los aspectos técnicos de las tecnologías digitales y reforzar su identidad y su sentido de empoderamiento 
(Bjørgen, 2010). Como método para integrar la instrucción de las competencias de ciudadanía y literacidad en TIC, esta investigación plantea actividades de aprendizaje en las que las personas estudiantes decodifican y crean medios digitales narrativos para presentar sus propias soluciones a los problemas de la comunidad.

\subsubsection{Pensamiento crítico, resolución de problemas y toma de decisiones}

El modelo ATC21s destaca la importancia del pensamiento crítico y la resolución de problemas. En concreto, promueve el uso adecuado del razonamiento deductivo e inductivo, la categorización y el análisis de información, la presentación de conclusiones basadas en dicho análisis, y la evaluación de argumentos y puntos de vista. Cabe mencionar que el propósito y estructura de las historias están estrechamente relacionados con estas habilidades. Todas las historias contienen argumentos implícitos o explícitos, ya sea a favor o en contra de algo (Gold et al., 2002).

La enseñanza de las habilidades de pensamiento crítico y resolución de problemas también desempeña un rol central en el desarrollo de la ciudadanía crítica. En Japón, los planes de estudio de primaria recomiendan el uso de organizadores gráficos de visualización de pensamiento en actividades de aprendizaje que impliquen la clasificación de información y el establecimiento de correlaciones entre eventos y hechos (Ministerio de Educación, Cultura, Deportes, Ciencia y Tecnología, 2018). La utilización de las herramientas de visualización de pensamiento se basa en la premisa de que las habilidades generales de pensamiento pueden enseñarse en el contexto del aula, transferirse entre distintos dominios de conocimiento y aplicarse a situaciones de la vida real.

Davies (2006) señala que la enseñanza del pensamiento crítico es más eficaz cuando habilidades generales de pensamiento se enseñan sistemáticamente en múltiples asignaturas escolares y se aplican a situaciones familiares. Este enfoque se describe como "infusión", y exige la instrucción explícita de las habilidades y disposiciones relacionadas con el pensamiento crítico mientras se tratan los contenidos específicos de las asignaturas (Ennis, 1989). Cabe señalar también que la adquisición de la competencia de pensamiento crítico requiere comprender y activar múltiples habilidades individuales, cada una de las cuales se relaciona con procesos cognitivos específicos, como la comparación, la clasificación, el análisis y la inferencia (Taizan, 2019). El uso de organizadores gráficos, impresos o digitales, puede apoyar la metacognición, permitiendo la optimización y visualización de estos procesos. En esta investigación, se propone el uso coordinado de organizadores gráficos, ampliamente 
utilizados en Japón, y recursos narrativos para apoyar el desarrollo del pensamiento crítico y la conciencia ciudadana.

\section{Metodología}

\subsection{Enfoque}

Esta investigación se organizó en cuatro etapas, y utilizó un abordaje mixto, integrando métodos cualitativos y cuantitativos para la recolección y procesamiento de la información. En la primera etapa, se realizaron tareas de diseño instruccional para desarrollar dos unidades de aprendizaje para cada país participante. En la creación de los recursos que apoyan estas unidades, se utilizó una taxonomía especializada para la clasificación y diseño de medios educativos dirigidos a apoyar el pensamiento crítico (Mena, 2020). Durante las actividades de aprendizaje, las personas estudiantes tuvieron la posibilidad de expresar sus habilidades de pensamiento crítico y su conciencia ciudadana a través de la creación de historietas. La naturaleza subjetiva y compleja de estas expresiones exige, en un primer nivel, un enfoque cualitativo. En concreto, se analizaron las historietas creadas por las personas estudiantes, para luego interpretar su estructura e intención. En un segundo nivel, se desarrollaron marcos de indicadores para asignar valores numéricos a los niveles de habilidad de pensamiento crítico y conciencia ciudadana expresados por las personas estudiantes en sus historias. La introducción de valores numéricos permitió la realización de análisis cuantitativos.

En la segunda etapa, se desarrollaron métodos de evaluación de pensamiento crítico y conciencia ciudadana mediante un abordaje narrativo (Frank, 2010), enfocado en el análisis de discurso y en el análisis textual. Algunas actividades de las unidades de aprendizaje, como el uso de organizadores gráficos, permiten a las personas docentes constatar cómo las personas estudiantes expresan sus ideas durante el proceso de instrucción, y en base a ello, las personas docentes pueden orientar el desempeño de las personas estudiantes. Estas interacciones pueden considerarse como ejemplos de evaluación formativa (Taras, 2005). Sin embargo, los métodos de evaluación propuestos en este estudio deben ser considerados como ejemplos de evaluación sumativa, ya que enfatizan los resultados del proceso de aprendizaje. Específicamente, estos métodos de evaluación buscan establecer cómo la participación de las personas estudiantes en las unidades de aprendizaje influye en sus expresiones de habilidades de pensamiento crítico y conciencia ciudadana.

En la tercera etapa, se implementaron las unidades de aprendizaje con el apoyo de las personas docentes a cargo de los grupos participantes. En esta etapa las personas estudiantes 
respondieron cuestionarios y crearon historietas. En la cuarta etapa se analizaron los cuestionarios e historietas, se les asignó puntajes, y los resultados fueron sometidos a un análisis estadístico descriptivo.

\subsection{Participantes}

En cada país se seleccionó una escuela pública de educación primaria, y en cada escuela se seleccionaron dos grupos de sexto año, incluyendo a un grupo experimental que participó en todas las actividades, y a un grupo de control que participó solo en los cuestionarios. En Costa Rica, participaron 26 estudiantes en el grupo experimental y 25 estudiantes en el grupo de control. En Japón, participaron 27 estudiantes tanto en el grupo experimental como en el grupo de control. Con el fin de minimizar las disparidades en las habilidades iniciales de las personas estudiantes, en ambos países se seleccionaron escuelas públicas, ubicadas en comunidades rurales, con uso limitado de computadoras y otros dispositivos electrónicos, y sin experiencias previas en la utilización de organizadores gráficos y en la creación de historietas. Adicionalmente, con el objetivo de posibilitar el establecimiento de grupos experimentales y de control, se seleccionaron escuelas primarias que contaran con al menos dos grupos de sexto grado.

La selección de estudiantes de sexto grado se basó en tres consideraciones fundamentales. La primera está relacionada con los resultados que pueden ser esperados en los procesos de instrucción de pensamiento crítico. El pensamiento crítico implica la transferencia de habilidades generales a distintos dominios de conocimiento, y requiere una instrucción sistemática y longitudinal. Esta instrucción debe introducirse en la educación primaria y ampliarse a la educación secundaria, adaptando las actividades de aprendizaje a los contenidos curriculares, y a las necesidades de las personas estudiantes (Higuchi, 2013, p. 340). Las unidades de aprendizaje propuestas en esta investigación ofrecen métodos idóneos para introducir la enseñanza de pensamiento crítico a nivel de primaria. Las dos consideraciones restantes se enfocan en la relación entre los procedimientos de las unidades de aprendizaje y el desarrollo cognitivo de las personas estudiantes. Al cumplir diez años, las personas experimentan cambios cruciales en su desarrollo, que les permiten comprender conceptos y situaciones más allá de sus propias experiencias personales. Los niños y niñas se vuelven capaces de aprender a partir de situaciones complejas, y de aplicar formas de pensamiento abstracto en actividades de mayor dificultad lingüística. También se vuelven capaces de hacer comparaciones, formular hipótesis y realizar correctamente operaciones de 
pensamiento lógico (Watanabe, 2011). Adicionalmente, al superar los 10 años, la mayoría de las personas adquiere la capacidad de comprender plenamente la naturaleza de la fantasía y la ficción, y cómo estas se representan en los medios narrativos (Adachi, 2012). En concreto, se vuelven capaces de distinguir entre los personajes de ficción y los de la vida real, y de reconocer el papel que desempeñan en las historias el montaje, los efectos especiales y otros recursos narrativos.

En ambos países, las personas directoras de los centros educativos y las personas estudiantes que participaron en las actividades brindaron su consentimiento informado al inicio de la primera unidad de aprendizaje, utilizando documentos homólogos, disponibles tanto en español como en japonés. Durante el proceso de investigación, se tomaron medidas para proteger la privacidad, integridad física, y la integridad emocional de las personas participantes, siguiendo los lineamientos del Comité de Ética de Investigación de la Universidad de Tsukuba.

Esta investigación define como sus unidades de análisis los objetos, digitales o físicos, creados o modificados por las personas estudiantes durante las unidades de aprendizaje. Estos objetos incluyen organizadores gráficos de visualización de pensamiento, guiones de historieta, historietas digitales y cuestionarios. Los métodos propuestos para la evaluación del pensamiento crítico y la conciencia ciudadana brindan especial atención al contenido de las historietas y cuestionarios.

\subsection{Técnicas de recolección}

La recolección de datos se realizó en una fase de instrucción y una fase de evaluación. En este apartado se detallan las tareas realizadas en cada una de ellas.

\subsubsection{Instrucción}

El primer objetivo de investigación se abordó mediante un proceso de instrucción desarrollado entre marzo y agosto del 2019 en Costa Rica, y entre julio de 2019 y enero de 2020 en Japón (Tabla 1). Durante el proceso de instrucción, las personas estudiantes miraron cortos animados que narran problemas relacionados con la escuela y la comunidad, usaron organizadores gráficos para expresar sus ideas, redactaron guiones, y utilizaron el software de edición de historietas Voicing Board para crear historietas que proponen soluciones a los problemas presentados en los cortos animados. 
Tabla 1. Actividades de las unidades de aprendizaje desarrolladas en el estudiantado, Costa Rica y Japón, 2019-2020

\begin{tabular}{|c|c|c|}
\hline Unidad & Actividad & Duración \\
\hline \multirow[t]{8}{*}{1} & Primera aplicación de cuestionario. & \multirow{8}{*}{$\begin{array}{l}7 \text { lecciones en } \\
\text { Costa Rica y en } \\
\text { Japón. }\end{array}$} \\
\hline & Visionado de corto animado sobre problemas de seguridad vial. & \\
\hline & Redacción de guion de historieta. & \\
\hline & Creación de historieta digital utilizando Voicing Board. & \\
\hline & Clasificación de personajes utilizando organizadores gráficos. & \\
\hline & Análisis de las causas del problema utilizando organizadores gráficos. & \\
\hline & Inferencia de soluciones utilizando organizadores gráficos. & \\
\hline & Evaluación de soluciones utilizando organizadores gráficos. & \\
\hline \multirow[t]{9}{*}{2} & Visionado de corto animado sobre problemas de basura. & \multirow{9}{*}{$\begin{array}{l}7 \quad \text { lecciones en } \\
\text { Costa Rica y } 6 \\
\text { lecciones } \\
\text { Japón. }\end{array}$} \\
\hline & Clasificación de personajes utilizando organizadores gráficos. & \\
\hline & Análisis de las causas del problema utilizando organizadores gráficos. & \\
\hline & Inferencia de soluciones utilizando organizadores gráficos. & \\
\hline & Redacción de guion de historieta. & \\
\hline & Creación de historieta digital utilizando software Voicing Board. & \\
\hline & Evaluación de soluciones utilizando organizadores gráficos. & \\
\hline & Votación estudiantil para elegir las soluciones más adecuadas. & \\
\hline & Segunda aplicación de cuestionario. & \\
\hline
\end{tabular}

Fuente: Elaboración del autor (2021).

Los cortos animados, que tratan problemas de seguridad vial y de tratamiento de desechos, fueron desarrollados utilizando el modelo de enseñanza a través de narrativa propuesto por Egan (1989). Según este modelo, las unidades de aprendizaje deben ser concebidas como buenas historias para ser contadas, destinadas a estimular la motivación y la imaginación de las personas estudiantes. Egan afirma que la introducción de la narrativa en la enseñanza debe guiarse por principios como el ritmo narrativo, los opuestos binarios y el significado afectivo. Mientras que el ritmo narrativo define cómo las historias introducen un problema y luego avanzan hacia la solución, los opuestos binarios se ocupan de los conflictos subyacentes. Estos conflictos suelen situar a los personajes en el centro de las luchas entre el bien y el mal, o entre la seguridad o el peligro. Al determinar los opuestos binarios relacionados con los temas que se abordan en el proceso de aprendizaje, se hace posible crear historias convincentes que funcionen como base para que las personas estudiantes reflexionen y creen a la vez sus propias historias. Además, al centrarse en las emociones e intenciones humanas, las historias pueden trascender los aspectos racionales y lógicos de un problema y transmitir formas de significado afectivo. De este modo, el uso de historias puede equilibrar los aspectos cognitivos y afectivos del aprendizaje.

Los cortos animados presentan a las personas estudiantes como protagonistas de historias que abordan problemas complejos que afectan tanto a las escuelas como a las 
comunidades. Las animaciones se estructuran en torno a varios opuestos binarios, como "buena ciudadanía" y "mala ciudadanía", y "bienestar" y "malestar". Estos recursos dan a entender que los "buenos ciudadanos y buenas ciudadanas" deben implicarse en la resolución de los problemas de la comunidad, para evitar el "malestar" y procurar el "bienestar", no sólo para ellos mismos sino también para todas las personas de su entorno. Las animaciones buscan que las y los estudiantes reconozcan estas estructuras implícitas y se motiven para participar en las actividades de aprendizaje.

Cabe señalar que los cortos animados ilustran situaciones que pueden considerarse parte habitual de la vida en las comunidades costarricenses y japonesas. En Costa Rica, podemos ver aceras estrechas, una señora que vende repostería por las calles, y lluvias intensas. En Japón, podemos ver arquitectura tradicional japonesa, caminos estrechos en zonas residenciales y un agricultor trabajando en un campo de arroz. Además, en la segunda unidad, las películas de animación muestran eventos celebrados en las comunidades donde se encuentran las escuelas participantes. Por ejemplo, en Costa Rica, los personajes llevan faroles hechos a mano a un parque por la noche, y desfilan en una banda musical al día siguiente, mientras participan en las celebraciones del Día de la Independencia. En Japón, los personajes se reúnen en el festival local de Daruma-ichi, en el que disfrutan comidas típicas y adquieren juguetes tradicionales.

\subsubsection{Evaluación}

En esta fase se construyeron dos métodos de evaluación complementarios para determinar los efectos de las actividades de aprendizaje en las habilidades de pensamiento crítico, en la conciencia ciudadana, y en las nociones de comunidad y participación ciudadana de las personas estudiantes. El primer método de evaluación consistió en el análisis de las historietas creadas por las personas estudiantes del grupo experimental. Este análisis se centró en expresiones de conciencia ciudadana y pensamiento crítico a través de narrativa.

El segundo método de evaluación consistió en un cuestionario aplicado antes de la primera unidad y al final de la segunda unidad, tanto en los grupos experimentales como en los grupos de control. Este cuestionario está conformado por cuatro preguntas abiertas, y pretende recoger información explícita e implícita sobre cómo los expresan sus opiniones sobre comunidad y ciudadanía. La primera pregunta del cuestionario trata de dilucidar si las personas estudiantes comprenden a las comunidades no sólo como lugares de residencia, sino también como grupos de personas que interactúan de diferentes maneras. La segunda 
pregunta examina el nivel de conocimiento expresado por las personas estudiantes sobre los problemas y condiciones de sus comunidades. Estas dos preguntas pretenden visualizar el escenario general en el que los niños sitúan las historias que crean mientras piensan en sus propias comunidades. La tercera y la cuarta pregunta se enfocan en las visiones de las personas estudiantes sobre la participación ciudadana, tanto en la vida escolar como en la vida comunitaria.

\subsection{Procesamiento de análisis}

Las historietas fueron evaluadas mediante un análisis de sus elementos microestructurales y macroestructurales. Mientras que la macroestructura se refiere a la organización jerárquica de los textos, la microestructura se ocupa de sus estructuras internas (Justice et al., 2006). Las acciones y los diálogos representados en cada panel de las historietas se sintetizaron en enunciados cortos que describen, individualmente, los elementos microestructurales de la historia. Cuando se colocan en una secuencia lineal, estos ofrecen también ofrecen una visión general de la macroestructura de la historia.

El análisis de las historietas se realizó en cada unidad de aprendizaje, siguiendo dos procedimientos distintos (Figura 2). El primer procedimiento pone el énfasis en la macroestructura de la historia y busca visualizar el nivel de aplicación de las habilidades de pensamiento crítico expresado en ella. Este procedimiento genera una medida denominada puntaje de pensamiento crítico (PPC), que tiene un rango de 1 a 4 puntos, y se estructura a partir de la relación de dos indicadores: conexión lógica entre los elementos de la historia, y linealidad (la cual se encuentra estrechamente relacionada con la coherencia narrativa). A las historietas que muestran conexión lógica y linealidad se les asigna un puntaje completo de 4 puntos. A las historietas que solo muestran conexión lógica se les asigna 3 puntos, y a las que solo muestran linealidad se les asigna 2 puntos. Por último, a las historietas que no reflejan ninguna de las variables se les asigna un PPC de 1 punto. 
Figura 2. Indicadores y puntuación para la evaluación de pensamiento crítico en historietas, Costa Rica y Japón, 2019-2020
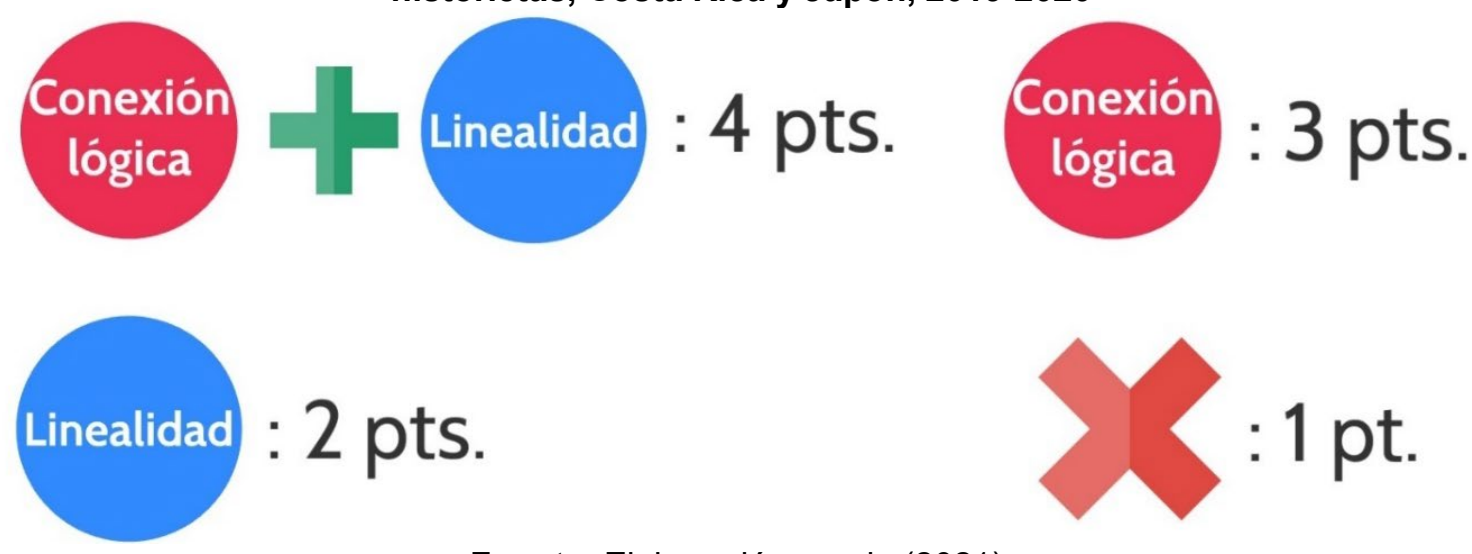

Fuente: Elaboración propia (2021).

El segundo procedimiento se centra en la microestructura de las historias, específicamente en las acciones individuales de los personajes representados en las historietas. En este análisis se excluyen los personajes de adultos y se incluyen solamente los personajes de estudiantes de edad escolar. En este procedimiento, se adapta la teoría de actantes propuesta por Propp (2015) para organizar a los personajes de las historietas en seis categorías, dependiendo de cómo se relacionan sus acciones con el problema y su solución. De este modo, se utilizan las contraposiciones de activo y pasivo, y de positivo y negativo para asignar a los personajes los actantes de Héroe, Ayudante, Destinador, Víctima, Falso Héroe y Villano (Figura 3). El objetivo de este procedimiento es evaluar el nivel de conciencia ciudadana de las personas estudiantes con base en cómo representan la iniciativa y la eficacia de los personajes de estudiantes incluidos en las historietas. La medida obtenida de este proceso se denomina puntaje de conciencia ciudadana (PCC), el cual también tiene un rango de 1 a 4 puntos.

La premisa del PCC es que las personas estudiantes que participan en las unidades de aprendizaje pueden utilizar a los personajes de estudiantes que incluyen en sus historietas para proyectar sus propias nociones de participación ciudadana. Por ejemplo, si en una historieta alguno de los personajes de estudiante trabaja por su propia iniciativa para solucionar el problema, ese personaje es considerado como un Héroe, que cumple un rol positivo y activo en la historia, por lo cual, a la historieta se le asigna un PCC completo de 4 puntos. Por otra parte, si uno de los personajes de estudiante es afectado por el problema, pero no toma ninguna acción para resolverla, es considerado como una Víctima en la historia, que cumple un rol negativo pero pasivo. Si en la historieta hay un personaje de Víctima y no 
hay otros actantes con puntajes más altos, se le asigna el puntaje correspondiente a Víctima, es decir, 2 puntos.

Figura 3. Categorías y puntuación para la evaluación de conciencia ciudadana en Activo historietas, Costa Rica y Japón, 2019-2020

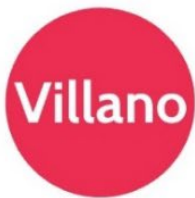

1 pt.

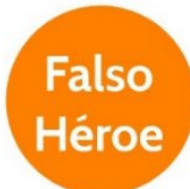

2 pts.
Pasivo
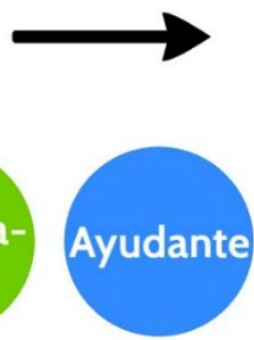

3 pts.
Activo

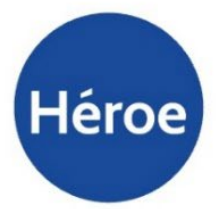

4 pts.
Problema Negativo

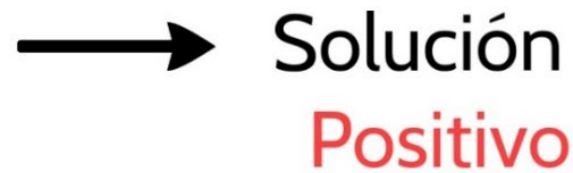

Fuente: Elaboración propia (2021).

Por otra parte, para la evaluación de cada pregunta del cuestionario se establecieron indicadores, y se definieron diferentes puntuaciones en función de la combinación de estos indicadores (Tabla 2), en una escala de 1 a 4 puntos. La puntuación media de las cuatro preguntas se presenta en una medida denominada puntaje total de cuestionario (PTC). Cabe mencionar que puntajes altos en la tercera (P3) y cuarta pregunta (P4) sugieren un alto nivel de conciencia ciudadana. En este sentido, los objetos de P3 y P4 están estrechamente relacionados con el objeto de PCC.

Con el fin de determinar los posibles efectos del proceso de instrucción en el desempeño de las y los estudiantes, se aplicaron distintos análisis estadísticos utilizando los programas $\mathrm{R}$ (versión 3.6.1) y RStudio (versión 1.4.1106). Primeramente, se realizaron pruebas de rango con signo de Wilcoxon pareadas para comparar los resultados obtenidos en el análisis de los comics y los cuestionarios. Asimismo, se utilizaron pruebas de rango con signo de Wilcoxon no pareadas para comparar el desempeño de las personas estudiantes de los dos países en cada unidad de aprendizaje. Las pruebas de rango con signo de Wilcoxon son pruebas no paramétricas que pueden utilizarse para comparar dos muestras relacionadas o medidas repetidas en la misma muestra (Wilcoxon, 1945). Estas pruebas se centran en las diferencias 
entre los rangos medios de las muestras, y se aplica en aquellos casos en los que las diferencias entre los valores medios de las muestras no tienen una distribución normal.

Tabla 2. Indicadores y puntuación para la evaluación de conciencia ciudadana en cuestionarios, Costa Rica y Japón, 2019-2020

\begin{tabular}{|c|c|c|c|}
\hline Pregunta & Objeto & Indicadores & Puntuación \\
\hline $\begin{array}{l}\text { P1. ¿Qué significa } \\
\text { comunidad? }\end{array}$ & $\begin{array}{l}\text { Definición de } \\
\text { comunidad }\end{array}$ & $\begin{array}{l}\text { A. Menciona su dimensión geográfica. } \\
\text { B. Menciona su dimensión social. } \\
\text { C. Describen cómo las personas se } \\
\text { relacionan entre sí o con el lugar donde } \\
\text { viven. }\end{array}$ & $\begin{array}{l}A+B+C: 4 \text { pts. } \\
A / B+C: 3 \text { pts. } \\
A+B: 2 \text { pts. } \\
\text { A/B: } 1 \text { pt. }\end{array}$ \\
\hline $\begin{array}{l}\text { P2. ¿Cuáles son las } \\
\text { características de } \\
\text { nuestra comunidad? }\end{array}$ & $\begin{array}{l}\text { Características } \\
\text { de comunidad }\end{array}$ & $\begin{array}{l}\text { A. Describe sus características geográficas. } \\
\text { B. Describe sus características sociales. } \\
\text { C. Describe problemas específicos de la } \\
\text { comunidad. }\end{array}$ & $\begin{array}{l}A+B+C: 4 \text { pts. } \\
A / B+C: 3 \text { pts. } \\
A+B: 2 \text { pts. } \\
\text { A/B: } 1 \text { pt. }\end{array}$ \\
\hline $\begin{array}{l}\text { P3. ¿Cuando hay un } \\
\text { problema en nuestra } \\
\text { escuela quién tiene } \\
\text { que resolverlo? ¿Por } \\
\text { qué? }\end{array}$ & $\begin{array}{l}\text { Participación y } \\
\text { conciencia } \\
\text { ciudadana }\end{array}$ & $\begin{array}{l}\text { A. Asigna roles positivos a los personajes. } \\
\text { B. Menciona valores positivos }{ }^{5} \text {. } \\
\text { C. Menciona valores negativos }{ }^{6} \text {. } \\
\text { D. Asigna roles negativos a los personajes. }\end{array}$ & $\begin{array}{l}\text { A+B: } 4 \text { pts. } \\
\text { A: } 3 \text { pts. } \\
\text { A+C: } 2 \text { pts } \\
\text { D: } 1 \text { pt. }\end{array}$ \\
\hline $\begin{array}{l}\text { P4. ¿Cuando hay un } \\
\text { problema en nuestra } \\
\text { comunidad quién } \\
\text { tiene que resolverlo? } \\
\text { ¿Por qué? }\end{array}$ & $\begin{array}{l}\text { Participación y } \\
\text { conciencia } \\
\text { ciudadana }\end{array}$ & $\begin{array}{l}\text { A. Asigna roles positivos a los personajes. } \\
\text { B. Menciona valores positivos. } \\
\text { C. Menciona valores negativos. } \\
\text { D. Asigna roles negativos a los personajes. }\end{array}$ & $\begin{array}{l}\text { A+B: } 4 \text { pts. } \\
\text { A: } 3 \text { pts. } \\
\text { A+C: } 2 \text { pts } \\
\text { D: } 1 \text { pt. }\end{array}$ \\
\hline
\end{tabular}

Fuente: Elaboración propia (2021).

Cabe mencionar que el conjunto de datos recogidos en esta investigación requiere la aplicación de pruebas no paramétricas debido a tres razones principales: el tamaño limitado de la muestra, la exclusión de los análisis de las personas estudiantes que no proporcionaron los datos necesarios, y la asimetría en la distribución de la mayoría de los valores incluidos en los análisis. Adicionalmente, en ambos países se utilizó el coeficiente de correlación de rangos de Spearman para determinar la correlación entre los puntajes de PPC, de PCC y los de las preguntas del cuestionario. Cabe señalar que el coeficiente de Spearman es un recurso estadístico no paramétrico que mide la fuerza de la asociación entre dos variables (Hauke y Kossowski, 2011), y que para el rechazo o aceptación del contraste de hipótesis estadísticas se asume un nivel de significancia del 5\%. Finalmente, los resultados de los análisis estadísticos se utilizaron para describir tendencias generales en el desempeño de las personas estudiantes.

\footnotetext{
5 Entusiasmo, colaboración, capacidad de diálogo, responsabilidad, empatía, agradecimiento, etc.

${ }^{6}$ Culpa, miedo, sentido de obligación, ganancia personal, falta de habilidad, etc.
} 


\section{Resultados}

Esta sección presenta los resultados de los procesos de instrucción y evaluación, aporta análisis estadísticos enfocados en los cambios y correlaciones de los resultados de evaluación, y ofrece interpretación y discusión sobre los resultados.

\subsection{Resultados del proceso de instrucción}

En esta subsección, se atiende del primer objetivo de investigación, mediante la descripción de las tendencias relevantes observadas en el proceso de instrucción. En ambos países, fue posible identificar dos tendencias principales. La primera tiene que ver con cuestiones procedimentales, y la aplicación del pensamiento crítico a la resolución de problemas. Especialmente en la primera unidad, algunos estudiantes mostraron dificultades para comprender los procedimientos de los organizadores gráficos. Las personas docentes respondieron oportunamente en la mayoría de los casos, orientando a las personas estudiantes. Esta situación se manifestó principalmente en las lecciones en las que se utilizaron organizadores gráficos para realizar análisis e inferencias, que pueden considerarse como las actividades de mayor carga cognitiva. A pesar de estas dificultades iniciales, la mayoría de estudiantes fueron capaces de analizar las causas del problema y construir guiones de historietas basados en las ideas que habían expresado en los organizadores gráficos.

Además, cuando se les dio la oportunidad de votar sobre las historietas creadas por sus pares, la mayoría de los participantes eligieron historias en las que los personajes de estudiantes desempeñaban un papel activo en la resolución de los problemas de la comunidad. Este resultado es especialmente destacable en Costa Rica, donde la mayoría de las personas estudiantes asignaron papeles pasivos a los niños en las preguntas del cuestionario relacionados con la participación ciudadana (P3 y P4). De este modo, las unidades de aprendizaje no sólo ayudaron a las personas estudiantes a establecer conexiones entre los acontecimientos concretos de la vida cotidiana y los principios abstractos del pensamiento crítico, sino que también les ayudaron a percibir el valor de las políticas y acciones basadas en la participación ciudadana activa.

La segunda tendencia está relacionada con la interacción de diferentes formas de discurso dentro de las unidades de aprendizaje. En algunos casos, las personas estudiantes aprovecharon las dos lecciones asignadas a la creación de historietas para ampliar las historias que habían planeado. Añadieron nuevos personajes y representaron situaciones más 
complejas que las que habían propuesto inicialmente. En consecuencia, algunas de las versiones finales de las historietas no se ajustaron completamente al contenido de los guiones. Estas discrepancias indican que las actividades de aprendizaje permiten que la creación narrativa sea un proceso flexible y beneficioso para el aprendizaje. Asimismo, el rendimiento de las personas estudiantes sugiere que el proceso de instrucción estableció con éxito un puente entre los elementos expositivos y descriptivos (organizadores gráficos y guiones) y los elementos narrativos (cortos animados e historietas) de las unidades de aprendizaje.

\subsection{Resultados del proceso de evaluación}

En esta subsección, se aborda el segundo objetivo de investigación, mediante la presentación de los hallazgos del análisis estadístico aplicado a los puntajes obtenidos por las personas estudiantes de ambos países.

\subsubsection{Cambios en el desempeño de los estudiantes}

Con el propósito de determinar los posibles efectos del proceso de aprendizaje en ambos países, se aplicaron pruebas de rango con signo de Wilcoxon pareadas para comparar los resultados obtenidos en las historietas y cuestionarios de cada unidad.

Tabla 3. Resultados de los estadísticos descriptivos en el grupo experimental de Costa Rica, 2019

\begin{tabular}{|c|c|c|c|c|c|c|c|c|c|c|c|c|c|c|}
\hline \multirow[b]{2}{*}{ Estadístico } & \multicolumn{7}{|c|}{ Unidad 1} & \multicolumn{7}{|c|}{ Unidad 2} \\
\hline & $\mathrm{PPC}^{7}$ & $\mathrm{PCC}^{8}$ & $\mathrm{P} 1^{9}$ & $\mathrm{P} 2^{10}$ & $P 3^{11}$ & $\mathrm{P} 4^{12}$ & $\begin{array}{l}\text { PTC } \\
13\end{array}$ & PPC & PCC & $\mathrm{P} 1$ & $\mathrm{P} 2$ & P3 & $\mathrm{P} 4$ & PTC \\
\hline$M^{14}$ & 2.00 & 2.83 & 2.07 & 1.94 & 2.00 & 2.29 & 8.00 & 2.78 & 3.50 & 2.64 & 1.75 & 2.07 & 1.43 & 7.93 \\
\hline$M D N^{15}$ & 1.50 & 3.00 & 2.00 & 2.00 & 1.00 & 2.00 & 8.00 & 3.00 & 4.00 & 2.00 & 2.00 & 1.00 & 1.00 & 7.50 \\
\hline$D S^{16}$ & 1.19 & 0.86 & 0.92 & 1.06 & 1.27 & 1.40 & 2.09 & 1.22 & 0.62 & 1.01 & 0.87 & 1.38 & 0.85 & 2.92 \\
\hline
\end{tabular}

Fuente: Elaboración propia (2021).

\footnotetext{
${ }^{7}$ Puntaje de pensamiento crítico.

8 Puntaje de conciencia ciudadana.

9 Puntaje de primera pregunta del cuestionario.

10 Puntaje de segunda pregunta del cuestionario.

11 Puntaje de tercera pregunta del cuestionario.

12 Puntaje de cuarta pregunta del cuestionario.

13 Puntaje total de cuestionario.

14 Media.

15 Mediana.

16 Desviación estándar.
} 
En el grupo experimental de Costa Rica, 18 estudiantes crearon ambas historietas y 15 completaron ambos cuestionarios. En Japón, 24 crearon ambas historietas y 23 estudiantes respondieron a ambos cuestionarios. En casos especiales, no se asignaron algunos de los puntajes. Por ejemplo, un participante de Japón elaboró ambas historietas, pero no incluyó personajes de estudiantes en la segunda. Por este motivo, no se asignaron valores PCC a este estudiante en la segunda unidad. Otro estudiante respondió a ambos cuestionarios, pero no contestó a una de las preguntas de la primera unidad.

Cabe señalar que solamente los datos de las personas estudiantes a los que se les asignaron puntuaciones en ambas unidades se incluyeron en las pruebas de rango con signo de Wilcoxon pareadas. En Costa Rica, se incluyeron 18 estudiantes en el análisis para PPC y PCC, 15 para $\mathrm{P} 1,13$ para $\mathrm{P} 2,15$ para $\mathrm{P} 3$ y 15 para $\mathrm{P} 4$. En Japón, se incluyeron 24 estudiantes en el análisis para la PPC, 23 para la PCC, 21 para el P1 y 23 para el P2, P3 y P4. Las tablas 3 y 4 muestran los puntajes de los grupos experimentales de cada país, y detallan los valores de la media, mediana y desviación estándar de los puntajes de los alumnos incluidos en las pruebas.

Tabla 4. Resultados de los estadísticos descriptivos en el grupo experimental de Japón,

\begin{tabular}{|c|c|c|c|c|c|c|c|c|c|c|c|c|c|c|}
\hline \multirow{2}{*}{ Estadístico } & \multicolumn{7}{|c|}{ Unidad 1} & \multicolumn{7}{|c|}{ Unidad 2} \\
\hline & PPC & PCC & $\mathrm{P} 1$ & P2 & P3 & P4 & PTC & PPC & PCC & $\mathrm{P} 1$ & P2 & P3 & $\mathrm{P} 4$ & PTC \\
\hline$M$ & 3.21 & 3.52 & 2.19 & 2.30 & 2.96 & 2.39 & 9.65 & 3.42 & 3.65 & 2.39 & 2.22 & 2.96 & 2.70 & 10.26 \\
\hline$M D N$ & 4.00 & 4.00 & 2.00 & 3.00 & 3.00 & 2.00 & 9.00 & 4.00 & 4.00 & 2.00 & 2.00 & 4.00 & 4.00 & 10.00 \\
\hline$D S$ & 1.14 & 0.79 & 1.17 & 1.26 & 1.15 & 1.27 & 3.17 & 1.10 & 0.57 & 1.03 & 1.17 & 1.40 & 1.46 & 2.82 \\
\hline
\end{tabular}

Fuente: Elaboración propia (2021).

En Costa Rica, los valores de PPC $\left(\mathrm{W}_{\text {pareado }}=6, \mathrm{p}<0,05\right)$ y PCC $\left(\mathrm{W}_{\text {pareado }}=2,5, \mathrm{p}<0,05\right)$ mostraron diferencias estadísticamente significativas entre las dos unidades. Por el contrario, no se observaron diferencias significativas en ninguna de las preguntas del cuestionario ni en el PTC, excepto en la pregunta 4, en la que los puntajes de los alumnos mostraron diferencias estadístiscamente significativas con entre las dos unidades $\left(W_{\text {pareado }}=39, p<0,05\right)$, con una media menor en la segunda unidad. En Japón, los resultados de la segunda unidad mostraron valores medios más altos en PPC y PCC, pero no se observaron diferencias significativas. Del mismo modo, los resultados de las preguntas del cuestionario y del PTC no mostraron diferencias significativas. 
En los grupos de control, 18 estudiantes completaron ambos cuestionarios en Costa Rica y 24 lo hicieron en Japón. Los resultados de estos cuestionarios también se analizaron mediante pruebas de rango con signo de Wilcoxon pareadas. En Costa Rica, no se observaron diferencias significativas en ningún puntaje. En Japón, sólo se observaron diferencias estadísticamente significativas en las puntuaciones de $\mathrm{P} 1\left(\mathrm{~W}_{\text {pareado }}=0, \mathrm{p}<0,05\right)$ y $\mathrm{P} 2\left(\mathrm{~W}_{\text {pareado }}=\right.$ $116, p<0,05)$.

\subsubsection{Comparación del desempeño de los estudiantes en Costa Rica y Japón}

Con el fin de comparar el desempeño de las personas estudiantes en ambos países, se realizaron varias pruebas de rango con signo de Wilcoxon, centradas en los puntajes de PPC y el PCC. Además, se incluyeron en el análisis los puntajes de P3 y P4, ya que se espera que estos dos valores complementen el puntaje de PCC, proporcionando información adicional sobre la conciencia ciudadana de las personas estudiantes. En estas pruebas se incluyeron únicamente las personas estudiantes incluidos en las pruebas mencionadas en la subsección anterior.

En la primera unidad de aprendizaje, el PPC $(\mathrm{W}=132, \mathrm{p}<0,05)$ de Japón mostró diferencias estadísticamente significativas con Costa Rica. También se observaron diferencias similares, que favorecieron los resultados de Japón, en PCC $(W=137, p<0,05)$, y en P3 $(W=149, p<0,05)$. En $P 4$, los participantes japoneses obtuvieron una media más alta, pero no se observaron diferencias estadísticamente significativas. En la segunda unidad, los resultados de Japón mostraron diferencias significativas con Costa Rica en PPC (W=174, $p<$ 0,05), $\mathrm{P} 3(\mathrm{~W}=156, \mathrm{p}<0,05)$ y $\mathrm{P} 4(\mathrm{~W}=160,5, \mathrm{p}<0,05)$. En PCC, los participantes japoneses obtuvieron una media más alta, pero no se observaron diferencias estadísticamente significativas.

\subsubsection{Correlaciones entre los puntajes de evaluación}

En ambas unidades, los resultados de ambos países mostraron una correlación positiva y significativa entre los puntajes de PPC y el PCC (Tabla 5). En otras palabras, las personas estudiantes con altas puntuaciones en PPC también obtuvieron altas puntuaciones en PCC. En Costa Rica, la correlación entre estos dos valores fue especialmente fuerte en la segunda unidad. En cuanto a las correlaciones entre los puntajes de las preguntas del cuestionario y PPC, y entre los puntajes de las preguntas del cuestionario y PCC, en Costa Rica los resultados mostraron correlaciones tanto negativas como positivas. Se encontraron 
correlaciones positivas significativas entre el P2 y el PPC en la primera unidad, y en la segunda unidad entre P1 y PPC, y entre P1 y PCC. En Japón, sólo se observaron correlaciones positivas, pero ninguna de ellas fue significativa.

Tabla 5. Correlaciones entre puntajes de evaluación de estudiantado, Costa Rica y Japón, 20192020

\begin{tabular}{|c|c|c|c|c|c|c|c|c|c|c|c|c|c|}
\hline \multirow[t]{2}{*}{ País } & & \multicolumn{6}{|c|}{ Unidad 1} & \multicolumn{6}{|c|}{ Unidad 2} \\
\hline & & PPC & PCC & P1 & P2 & P3 & P4 & PPC & PCC & P1 & P2 & P3 & P4 \\
\hline \multirow{5}{*}{$\begin{array}{l}\text { Costa } \\
\text { Rica }\end{array}$} & PCC & $0.48^{*}$ & & & & & & $0.72^{*}$ & & & & & \\
\hline & $\mathrm{P} 1$ & 0.39 & -0.02 & & & & & $0.54^{*}$ & $0.54^{*}$ & & & & \\
\hline & P2 & $0.53^{*}$ & 0.38 & -0.02 & & & & -0.20 & 0.11 & -0.07 & & & \\
\hline & P3 & -0.42 & -0.01 & -0.49 & -0.24 & & & -0.18 & 0.04 & 0.13 & 0.02 & & \\
\hline & $\mathrm{P} 4$ & 0.32 & 0.30 & 0.02 & 0.32 & 0.12 & 1 & -0.26 & 0.15 & 0.01 & 0.34 & 0.47 & 1 \\
\hline \multirow{5}{*}{ Japón } & $\mathrm{PCC}$ & $0.50^{*}$ & & & & & & $0.51^{*}$ & & & & & \\
\hline & $\mathrm{P} 1$ & 0.31 & 0.40 & & & & & 0.29 & 0.16 & & & & \\
\hline & P2 & 0.29 & 0.15 & 0.35 & & & & 0.12 & 0.15 & 0.40 & & & \\
\hline & P3 & 0.24 & 0.02 & 0.08 & 0.38 & & & 0.21 & 0.12 & 0.15 & -0.18 & & \\
\hline & $\mathrm{P} 4$ & 0.18 & 0.30 & 0.23 & 0.10 & 0.45 & 1 & 0.25 & 0.14 & 0.06 & -0.38 & 0.41 & 1 \\
\hline
\end{tabular}

* Correlación positiva y significativa, $p<0.05$.

Fuente: Elaboración propia (2021).

\subsection{Discusión de resultados}

En esta investigación, se esperaba que, como resultado del proceso de instrucción, los grupos experimentales obtuvieran puntuaciones significativamente más altas en los cuestionarios de la segunda unidad que los grupos de control. Asimismo, se esperaba que los grupos de control no mostraran diferencias significativas entre las puntuaciones de las dos unidades. Sin embargo, estas tendencias no se observaron. La ausencia de diferencias significativas en los resultados de los cuestionarios de los grupos experimentales podría sugerir que las unidades de aprendizaje tuvieron un efecto limitado en la forma en que las personas estudiantes comprenden los conceptos y las características de las comunidades, y en la forma en que ven la ciudadanía a través de su propio rol en la solución de problemas en la escuela y la comunidad. Además, algunos cambios en los resultados de los grupos de control podrían apuntar a factores individuales que afectaron el rendimiento de los participantes. Por ejemplo, los incrementos significativos en las puntuaciones de dos preguntas en el grupo de control japonés, pueden estar mostrando que, entre las dos recopilaciones de datos este grupo recibió algún tipo de instrucción que amplió efectivamente sus visiones sobre comunidad y la ciudadanía.

Aunque no reflejan las tendencias esperadas originalmente, los resultados del cuestionario cobran importancia cuando se contrastan con las ideas y actitudes expresadas 
por las personas estudiantes en las historietas. Los resultados del análisis de las historietas muestran tendencias diferentes en los dos países participantes. Un desfase entre los niveles iniciales de rendimiento en ambos países podría explicar estas disparidades. En Japón, la mayoría de estudiantes logró obtener puntuaciones altas en la primera unidad. Así, aunque se observaron ligeros incrementos en la segunda unidad, los buenos resultados de la primera pueden haber reducido el espacio para que los participantes aumentaran sus puntuaciones en la segunda unidad. De este modo, se podría argumentar que las unidades de aprendizaje tuvieron un impacto limitado sobre el nivel de conciencia ciudadana y las habilidades de pensamiento crítico de los participantes japoneses. Para muchos niños y niñas de Japón, las unidades de aprendizaje parecen haber servido fundamentalmente como una oportunidad para expresar sus actitudes y habilidades de forma novedosa. Por otro lado, en Costa Rica, donde los resultados de la primera unidad fueron inferiores a los de Japón, muchos niños lograron aumentar sus puntuaciones en la segunda unidad. Esta tendencia sugiere que, en entornos en los que los participantes tienen limitaciones iniciales, la creación de historias puede ayudar a potenciar su pensamiento crítico y su conciencia ciudadana.

Investigaciones previas ofrecen perspectivas valiosas sobre cómo los niños de diferentes contextos culturales expresan sus ideas a través de la narrativa. Watanabe (2004) determinó que, cuando se les pide que describan una historia representada en una historieta de cuatro paneles, los niños japoneses tienden a organizar sus ideas principalmente en estructuras lineales e inductivas, en contraste con los niños estadounidenses, quienes tienden a resaltar la conclusión de la historia mientras utilizan mayoritariamente patrones deductivos. Este estudio no incluye los patrones narrativos de los niños costarricenses, pero podría argumentarse que dichos patrones son más cercanos a los patrones de los niños estadounidenses, ya que ambos países comparten entornos mediáticos similares y utilizan idiomas con estructuras gramaticales cercanas. Teniendo en cuenta que la presentación lineal de las acciones está relacionada con uno de los indicadores del puntaje de PPC, la prevalencia de estas estructuras narrativas en la cultura japonesa puede haber influenciado los resultados de la evaluación, generando puntajes más altos en Japón.

Procedimientos de comunicación recurrentes relacionados con condiciones culturales también podrían estar afectando los resultados de la evaluación. Hall (1976, p. 91) introdujo el concepto de comunicación de alto y bajo contexto para explicar cómo la cultura afecta la forma en que los individuos codifican, transmiten e interpretan la información. En los mensajes de alto contexto, la mayor parte de la información se encuentra en el contexto físico o ya ha 
sido interiorizada por los individuos que participan en el proceso de comunicación. En otras palabras, sólo una pequeña parte de la información está codificada en el mensaje explícito. Por otro lado, la comunicación de bajo contexto requiere que la mayor parte de la información sea explícita en el mensaje codificado. La comunicación de alto contexto se apoya en procesos de programación, a través de los cuales los individuos aprenden a evaluar la información no verbal y a reconocer las intenciones, deseos y motivaciones de otras personas. De este modo, la comunicación de alto contexto puede ser más eficaz y satisfactoria. También puede permitir que múltiples individuos produzcan mensajes considerablemente unificados. Cabe señalar que mientras que la comunicación de alto contexto predomina en países como Alemania y Japón, la comunicación de bajo contexto es frecuente en países como Estados Unidos de América y Costa Rica.

El estilo de comunicación predominante en un país influye en la forma en que docentes y estudiantes interactúan en el aula. En consecuencia, cuando participan en secuencias complejas de actividades de aprendizaje, en las que se espera que infusionen valores de buena ciudadanía en sus propias historias, los niños japoneses pueden tener otra ventaja sobre sus pares costarricenses. La programación cultural de las escuelas japonesas puede proporcionar a las personas estudiantes la capacidad para seguir instrucciones complejas y deducir qué valores e ideas son más deseables para ser expresados en el aula. Por el contrario, la predominancia de un estilo de comunicación de bajo contexto podría estar impidiendo a las personas estudiantes costarricenses reconocer los elementos implícitos de los mensajes proporcionados durante las actividades de aprendizaje. Asimismo, debido a que tiende a producir mensajes más flexibles, la comunicación de bajo contexto podría estar condicionando a los niños costarricenses a crear historias más heterogéneas, y muchas de esas historias podrían no coincidir con los criterios para la evaluación de conciencia ciudadana utilizados en el análisis de historietas. 
Figura 4. Ejemplo de contraste en cuestionario e historieta en Costa Rica

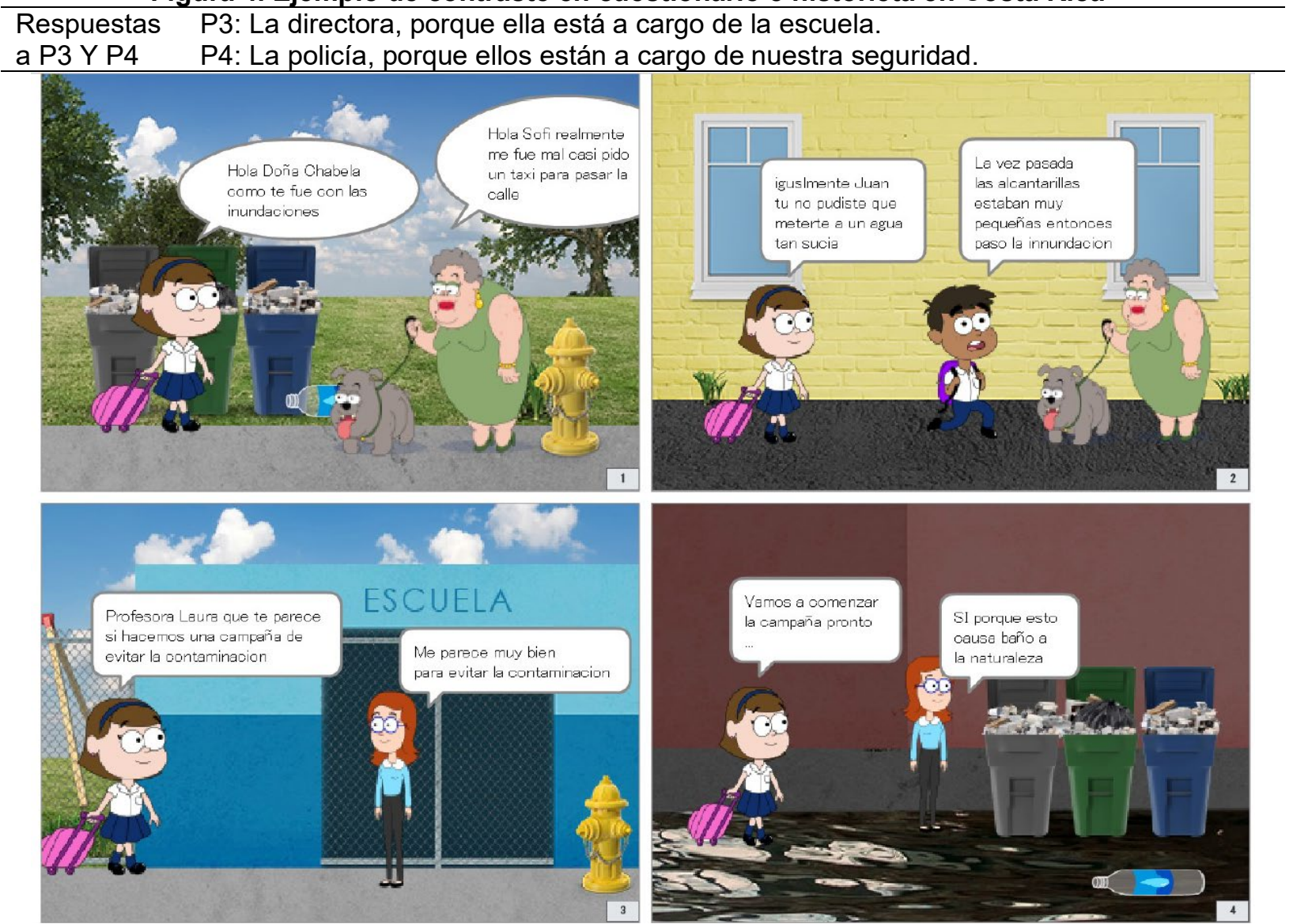

Fuente: Elaboración propia con base en productos creados por estudiantes (2021).

Por último, la explicación de por qué las historietas y los cuestionarios arrojan resultados diferentes a pesar de abordar conceptos y actitudes afines puede encontrarse en relaciones entre desarrollo cognitivo y discurso. Algunos de los participantes que respondieron en los cuestionarios que los docentes, directores y alcaldes deberían resolver los problemas de las escuelas y las comunidades, también crearon historias que mostraban a los niños y a otros ciudadanos participando activa y eficazmente en la resolución de dichos problemas. Por ejemplo, en Costa Rica, una historieta muestra a dos estudiantes cooperando para solucionar los problemas de basura en su ciudad (Figura 4). En el primer cuadro, una de las estudiantes conversa con una señora sobre los problemas de inundaciones en la comunidad. En el segundo cuadro, un estudiante se une a la conversación y hace notar que las inundaciones están relacionadas con bloqueos en los sistemas de alcantarillado. En el tercer cuadro, la estudiante se encuentra en la escuela y acuerda con su maestra iniciar una campaña para 
limpiar la comunidad, y en el cuarto cuadro, consolidan su compromiso mientras observan las calles inundadas y la basura acumulada.

En Japón, una de las historietas representa a dos estudiantes que también se organizan para abordar el problema de la basura (Figura 5). En el primer cuadro, dos estudiantes se encuentran el festival local. En el segundo cuadro, notan que la basura se está acumulando, y discuten sobre solicitarle a los trabajadores municipales que instalan más basureros. En el tercer cuadro, llevan su solicitud a uno de los trabajadores, y en el cuarto cuadro, se encuentran satisfechos pues ya se han instalado nuevos basureros, y el campo del festival está limpio.

Figura 5. Ejemplo de contraste en cuestionario e historieta en Japón

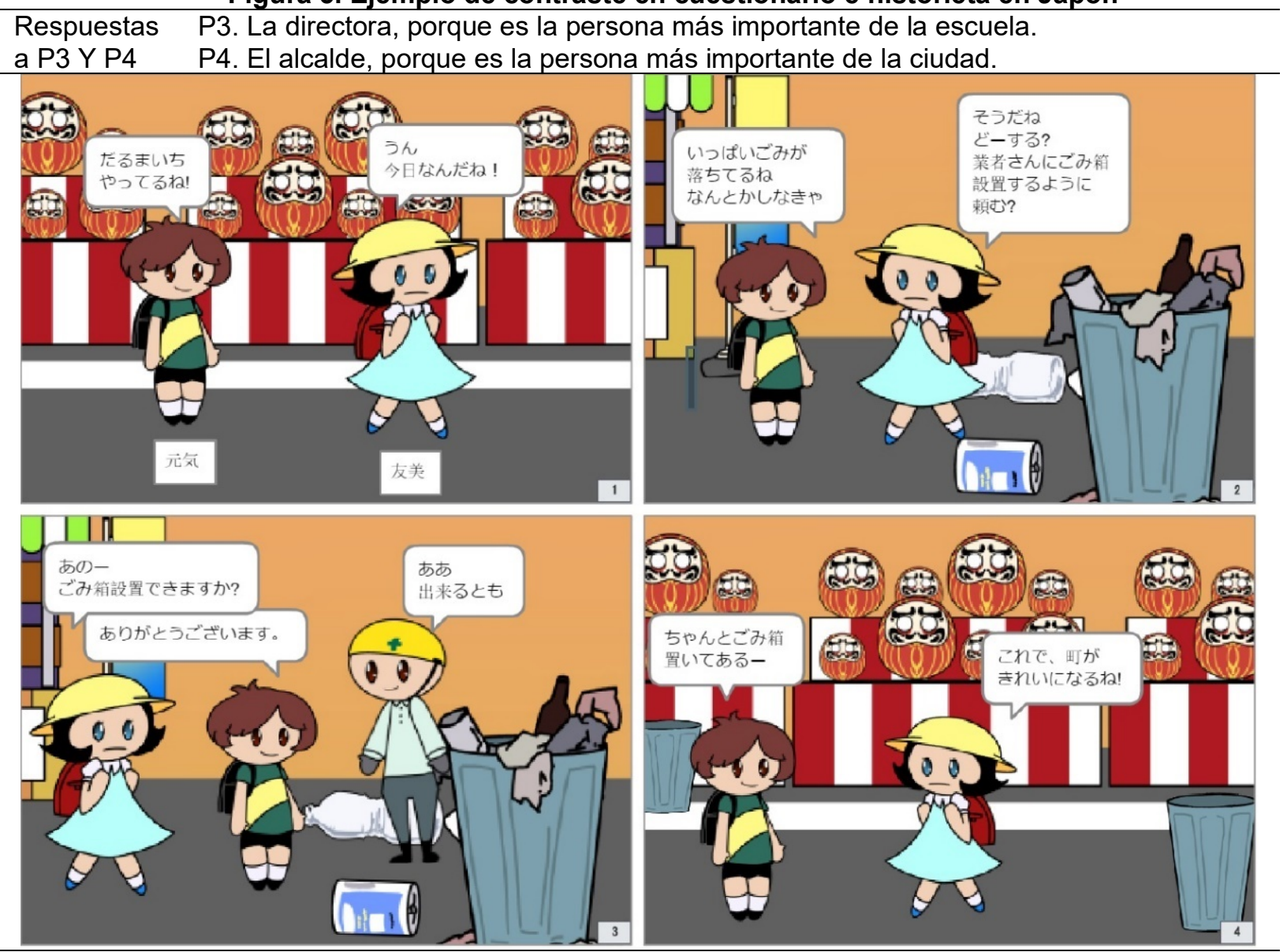

Fuente: Elaboración propia con base en productos creados por estudiantes (2021).

Al observar estos contrastes entre las respuestas a las preguntas del cuestionario y el contenido de las historietas, se puede afirmar que algunos estudiantes se sienten seguros al expresar una ciudadanía positiva y activa a través de la narrativa, pero tienen dificultades para 
hacerlo usando textos descriptivos y expositivos. Este hallazgo sugiere que, al combinar la instrucción de múltiples competencias, especialmente con actividades de aprendizaje destinadas a influir en la forma en que los individuos ven su propio rol en la comunidad, las personas educadoras deberían considerar la implementación de formas de instrucción y evaluación sustentadas en la narrativa.

\section{Conclusiones}

En esta investigación, se desarrollaron dos unidades de aprendizaje que integran recursos narrativos y organizadores gráficos para apoyar el desarrollo de múltiples competencias ATC21s en escuelas primaras de Costa Rica y Japón. Adicionalmente, múltiples métodos de evaluación, enfocados en el pensamiento crítico (PPC) y la conciencia ciudadana (PCC) expresada por las personas estudiantes mediante distintas formas de discurso, fueron propuestos para determinar los resultados del proceso de aprendizaje. Los principales resultados de la aplicación de estos métodos de evaluación pueden ser resumidos en los siguientes cuatro puntos.

1. Como resultado de la participación en las unidades de aprendizaje, la mayoría de las personas estudiantes pudieron aumentar sus puntajes de PPC y PCC. Sin embargo, estas diferencias de rendimiento sólo fueron significativas en Costa Rica.

2. En un sentido general, las personas estudiantes japonesas demostraron un mejor desempeño que las costarricenses en PPC, PCC y en varias preguntas del cuestionario.

3. En ambos países, se observaron correlaciones significativas entre las puntuaciones del PPC y del PCC, sin embargo, no se observaron correlaciones significativas entre las puntuaciones de las preguntas del cuestionario y las puntuaciones de PPC y PCC.

4. Es posible que las personas estudiantes sean capaces de expresar su conciencia ciudadana con mayor comodidad cuando utilizan textos narrativos, como las historietas, que cuando utilizan textos descriptivos y explicativos, predominantes en los cuestionarios.

Al observar los resultados de esta investigación, es importante tomar en consideración sus limitaciones. Una primera limitación se relaciona con la especificidad y el alcance de dichos resultados. Cualquier método de evaluación solo ofrece información fiable sobre el rendimiento de las personas estudiantes en el contexto específico de las experiencias de aprendizaje que se evalúan. Si algún estudiante no consigue aplicar determinadas destrezas y actitudes 
durante una actividad de aprendizaje, no significa que carezca intrínsecamente de la capacidad para hacerlo en otros contextos de aprendizaje o en situaciones de la vida real. Este principio se aplica a los resultados presentados en esta investigación. En tal sentido, estos resultados no deben considerarse una representación de las capacidades de todas las personas estudiantes de ninguno de los países participantes, ni un retrato definitivo de las capacidades específicas de las personas participantes en el estudio. Los resultados pueden ser diferentes en distintos centros educativos, y se puede esperar que cualquier estudiante desarrolle sus competencias mediante una instrucción sistemática y precisa.

Una segunda limitación se manifiesta en el uso extensivo de tiempo, recursos didácticos y recurso humano durante la investigación, y en la dificultad de reproducir estas condiciones en las escuelas de Costa Rica y Japón. La implementación de las unidades de aprendizaje requirió no solo la coordinación minuciosa con las personas docentes a cargo de los grupos experimentales, sino también el trabajo técnico y artístico de estudiantes universitarios, que en ambos países, contribuyeron en la elaboración de los cortos animados. Adicionalmente, la evaluación de las historietas y cuestionarios, y los análisis estadísticos de los datos resultantes, requirieron los aportes de seis personas investigadoras. En este sentido, las posibilidades para reproducir experiencias homólogas en múltiples centros de enseñanza, que permitan la comparación y profundización de los resultados, son limitadas.

Una tercera limitación puede identificarse en la naturaleza situada de las experiencias de aprendizaje realizadas durante la investigación. Las actividades de instrucción y evaluación, como cualquier otra acción realizada en entornos sociales, pueden verse influenciadas por factores sistémicos y coyunturales. Por ejemplo, diferencias en las condiciones específicas de centros educativos, en el sistema escolar y en la cultura política de países, regiones y comunidades pueden generar sesgos en los resultados de las actividades de aprendizaje.

A pesar de estas limitaciones, los métodos de evaluación propuestos en esta investigación pueden ser adoptados en distintos contextos, y aportar información útil para conocer el estado actual y la evolución de las habilidades de pensamiento crítico y la conciencia ciudadana en grupos específicos de estudiantes. También pueden proporcionar información sobre cómo el rendimiento de un grupo específico puede variar en relación con el rendimiento de otros grupos. De manera general, el valor de este estudio reside en la contribución que puede hacer a los debates sobre cómo enseñar y evaluar competencias múltiples en la educación primaria, y sobre cómo la integración de diferentes métodos de 
evaluación puede ofrecer información relevante sobre formas eficaces de apoyar y transformar la educación ciudadana en las aulas de diversos países.

Por otro lado, con base en los resultados de esta investigación, es posible extender la integración de la narrativa en el desarrollo del pensamiento crítico y la conciencia ciudadana a otros marcos de competencias. En concreto, esta tarea puede abordarse mediante una adaptación de dichos métodos a los indicadores de marcos de competencia emergentes. En el 2019, se presentó la Brújula del Aprendizaje 2030 (Organisation for Economic Co-operation and Development, 2019), el cual comparte algunos elementos clave con el marco ATC21s. Este nuevo modelo propone la enseñanza de competencias, compuestas por conocimientos, habilidades, actitudes y valores, para ayudar a las personas estudiantes a actuar independientemente mediante el establecimiento de objetivos, y la actuación responsable para lograr un cambio positivo en sus comunidades. Por ejemplo, incluye las literacidades digitales y de datos como uno de sus fundamentos básicos, las cuales están estrechamente relacionados con la Literacidad en TIC del ATC21s.

Asimismo, en sus competencias transformadoras, la Brújula de Aprendizaje 2030 incorpora la conciliación de tensiones y dilemas, y la toma de responsabilidades. Estas competencias desempeñan un papel crucial en el desarrollo de la conciencia ciudadana y la participación ciudadana en las comunidades locales y globales. Adicionalmente, ubica la anticipación, la acción y la reflexión en el centro del proceso de aprendizaje, creando condiciones favorables para el desarrollo del pensamiento lógico y reflexivo, los cuáles se relacionan estrechamente con el pensamiento crítico, la resolución de problemas y la toma de decisiones del modelo ATC21s. Debido a su énfasis en la resolución de problemas de la comunidad, la Brújula de Aprendizaje 2030 puede considerarse como un marco idóneo para abordar la instrucción y la evaluación del pensamiento crítico y la conciencia ciudadana. De este modo, en el futuro, la implementación de unidades de aprendizaje cercanas a la metodología propuesta en esta investigación debería incorporar ajustes que reflejen las competencias de la Brújula de Aprendizaje de manera explícita y extensa.

Finalmente, es importante reflexionar sobre las tareas pendientes en la implementación de metodologías de instrucción y evaluación transversal de competencias en los centros educativos de Costa Rica. Desde principios de siglo, las Competencias Clave han guiado el diseño de las pruebas PISA, las cuales se han convertido en la principal herramienta para visibilizar y comparar el desempeño de los sistemas educativos en distintos países (Rychen y Salganik, 2003). El modelo ATC21s y la Brújula de Aprendizaje 2030 han aportado nuevos 
abordajes al aprendizaje basado en competencias, y fortalecido el consenso sobre la necesidad de desarrollar e implementar nuevas formas de evaluación que puedan ser adaptadas a distintas realidades geográficas y culturales. Estas tendencias apuntan a un esfuerzo concertado para crear un estándar educativo internacional, que favorezcan el intercambio global de talento, ideas y experiencias, mediante el aprendizaje y evaluación de competencias.

En esta coyuntura, el sistema educativo de Costa Rica está llamado a impulsar cambios estructurales en tres ámbitos intrínsicamente relacionados. Primero, es imperativo fortalecer el rol del diseño instruccional en la formación y práctica docente, de manera que cada persona docente cuente con los conocimientos conceptuales y procedimentales necesarios para extraer indicadores de los distintos marcos de competencias, vincularlos con los contenidos de las distintas asignaturas, e infusionarlos en actividades de aprendizaje y evaluación. De esta manera, las personas docentes pueden encontrar soluciones a problemas comunes, compartir sus experiencias, y convertirse en agentes de cambio en sus centros educativos. Segundo, se deben promocionar métodos de instrucción que favorezcan la inclusión educativa, reconociendo la diversidad del estudiantado, e implementando actividades de aprendizaje que fomenten la expresión individual de cada estudiante (Fernández, 2013). La implementación de estos métodos puede crear las condiciones propicias para evaluar el nivel de dominio de cada estudiante sobre las distintas competencias, y para diseñar medidas de seguimiento dirigidas a la reducción de brechas de aprendizaje. El tercer ámbito de acción involucra la ampliación y promoción de repositorios digitales de recursos audiovisuales, recursos informáticos y guías de actividad de aprendizaje, que puedan servir de andamio en la implementación de actividades dirigidas al desarrollo de competencias. Estos repositorios pueden tomar como referencia plataformas creadas para el mismo propósito en países con sistemas escolares enfocados en el aprendizaje de competencias, y ser enriquecidos y expandidos sostenidamente con los recursos creados por las personas docentes.

\section{Agradecimientos}

Esta investigación fue realizada con el apoyo financiero y logístico de la Oficina de Asuntos Internacionales y Cooperación Externa de la Universidad de Costa Rica, y del Ministerio de Educación, Cultura, Deportes, Ciencia y Tecnología de Japón. Los análisis cualitativos fueron realizados con el apoyo de Rodrigo Muñoz, Nicole Chaves, Shunya Koga y Shingo Fujii. El análisis estadístico fue realizado con el apoyo de Melissa Valverde. 


\section{Referencias}

Adachi, Emi. (2012). La comprensión de los niños sobre la realidad de los personajes de la televisión: A partir de entrevistas a niños de entre 6 y 9 años [Publicado en japonés]. Revista de la asociación japonesa de investigación en ciencias psicológicas, 33(1),16245. https://doi.org/10.20789/jraps.33.1 16

Anwal, Nafisa., and Griffin, Patrick. (2018). Design considerations for 21st century educational assessments. European Conference on Games Based Learning, 15. Recuperado de https://bit.ly/3w2GVAd

Bjørgen, Anne Mette. (2010). Boundary crossing and learning identities - Digital storytelling in primary schools. Seminar.Net, 6(2). Recuperado de https://bit.ly/2Xh5V8W

Brewer, William F. (1995). To Assert that all human knowledge is represented in terms of stories is certainly wrong. En Robert S. Wyer (Ed.), Knowledge and memory: The real story. Advances in social cognition (pp. 211-225). Lawrence Erlbaum.

Care, Ester., Scoular, Claire., and Awwal, Nafisa. (2016). Assessing collaborative skills through games. The $10^{\text {th }}$ European Conference on Games Based Learning, 6-7 october, pp. 118122. https://bit.ly/362c24s

Cisco. (2010). Assessment and Teaching of 21st Century Skills: Status Report as of January 2010. https://bit.ly/3o1x5wk

Davies, W. Martin. (2006). An 'infusion' approach to critical thinking: Moore on the critical thinking debate. Higher Education Research \& Development, 25(2), 179-193. https://doi.org/10.1080/07294360600610420

Egan, Kieran. (1989). Teaching as storytelling: an alternative approach to teaching and the curriculum. University of Chicago Press.

Ennis, Robert H. (1989). Critical thinking and subject specificity: Clarification and needed research. Educational https://doi.org/10.3102/0013189X018003004

Fernández, José María. (2013). Competencias docentes y educación inclusiva. Revista $\begin{array}{llll}\text { Electrónica De Investigación } \quad \text { Educativa, } & \text { 15(2), }\end{array}$ http://redie.uabc.mx/vol15no2/contenido-fdzbatanero.html

Frank, Arthur W. (2010). Letting stories breathe: A socio-narratology. Chicago IL.: University of Chicago Press.

Freire, Paulo. (2017). Pedagogía del oprimido. Madrid: Siglo Veintiuno Editores.

Fundación Japón. (2019). Informe sobre la 20ª encuesta de sensibilización de los jóvenes de 18 años: Conciencia sobre la sociedad y la nación. Versión resumida. [Publicado en japonés]. Recuperado de https://bit.ly/3cShxpK 
Giroux, Henry A. (1989). Schooling for democracy: Critical pedagogy in the modern age. Routledge.

Gold, Jeff., Holman, David., and Thorpe, Richard. (2002). The role of argument analysis and storytelling in facilitating critical thinking. Management Learning, 33(3), 371-388. https://doi.org/10.1177/1350507602333005

Griffin, Patrick., McGaw, Barry., and Care, Esther. (2012). Assessment and Teaching of 21 st Century Skills. Springer. https://link.springer.com/book/10.1007/978-94-007-2324-5

Hall, Edward T. (1976). Beyond Culture. Garden City, NY: Anchor Press.

Hauke, Jan., and Kossowski, Tomasz. (2011). Comparison of values of Pearson's and Spearman's correlation coefficients on the same sets of data. Quaestiones Geographicae, 30(2), 87-93. https://doi.org/10.2478/v10117-011-0021-1

Higuchi, Naohiro. (2013). Teoría y práctica de la instrucción del pensamiento crítico: Métodos para la instrucción de habilidades de Pensamiento crítico en América y adaptaciones a las lecciones de estudios integrados en Japón [Publicado en japonés]. Tokio: Gakubunsha. Disponible en https://bit.ly/35XvJKK

Ikeda, Miyako., and García, Emma. (2014). Grade repetition: A comparative study of academic and non-academic consequences. OECD Journal: Economic Studies, 2013(1). https://doi.org/10.1787/eco studies-2013-5k3w65mx3hnx

Justice, Laura M., Bowles, Ryan P., Kaderavek, Joan N., Ukrainetz, Teresa A., Eisenberg, Sarita L, and Gillam, Ronald B. (2006). The index of narrative microstructure: A clinical tool for analyzing school-age children's narrative performances. American Journal of Speech-Language Pathology, 15(2), 177-191. https://doi.org/10.1044/1058$\underline{0360(2006 / 017}$

Kamiya, Yoshio. (2014). MOOC y habilidades del siglo XX: Nuevo despliegue de las TIC en la educación [Publicado en japonés]. Revista de la Universidad y Colegio Aichi Gakusen, (49), 43-48. Recuperado de https://bit.ly/2SGuRHq

Karaki, Kiyoshi. (2016). ¿Qué son las competencias cívicas? Indagaciones sobre el pasado, presente y futuro de la educación en estudios sociales [Publicado en japonés]. Toyokan. https://doi.org/10.15068/00155402

Koike, Kenji. (2019). Un estudio del aprendizaje sobre la educación artística utilizando el programa de años intermedios del bachillerato internacional: Con un enfoque en la teoría de las habilidades ATL (enfoques de las habilidades de aprendizaje) [Publicado en japonés]. Estudios en educación del arte, 51(1), 161-168. https://doi.org/10.19008/uaesj.51.161

Korsgaard, Morten Timmermann., Larsen, Vibe., and Wiberg, Merete. (2020). Thinking and researching inclusive education without a banister - visiting, listening and tact as a foundation for collective research on inclusive education. International Journal of Inclusive Education, 24(5), 496-512. https://doi.org/10.1080/13603116.2018.1469680 
Lam, Kee-Fui Turner, Wang, Tzu-Hua, Vun, Yee-Shih and Ku, Ning. (2020). Using DDMT teaching model to cultivate critical thinking in a STEAM classroom. In Klinge VillalbaCondori, Agustín Aduriz-Bravo, Jari Lavonen, Lung-Hsiang Wong, and Tzu-Hua Wang (Eds.), Education and Technology Sciences, (pp. 47-57). https://bit.ly/3jodZ32

Loría, Miguel., y Martínez, Josué. (2018). La educación pública costarricense: principales tendencias y desafíos. San José: La Academia de Centroamérica. https://bit.ly/3AehvTJ

Mena Araya, Aarón Elí. (2020). Una taxonomía de medios educativos para el desarrollo del pensamiento crítico: Dominios de acción y tipologías textuales, Estudios Pedagógicos, 46(1), 203-222. https://doi.org/10.4067/S0718-07052020000100203

Ministerio de Educación, Cultura, Deportes, Ciencia y Tecnología. (2018). Uso y evaluación cualitativa de las TIC para el aprendizaje activo e independiente, dialógico y activo: $A$ partir de las experiencias de las escuelas TIC en 2017 [Publicado en japonés]. https://bit.ly/3dgVgR9

Murata, Yokuo., and Yamaguchi, Mitsuru. (Eds.) (2010). Education in contemporary Japan System and content. Tokyo: Toshindo.

Organization for Economic Co-operation and Development. (2019). OECD Future of Education and Skills 2030 Conceptual learning framework: Core Foundations for 2030. Recuperado de https://www.oecd.org/education/2030-project/teaching-and-learning/learning/corefoundations/Core Foundations for 2030 concept note.pdf

Oyanagi, Wakio. (2016). Un informe de estudio sobre el método de investigación del programa nacional de evaluación de las TIC en Australia [Publicado en japonés]. Revista japonesa de tecnología educativa, 40(3), 209-220. https://doi.org/10.15077/jjet.40036

Portillo Torres, Mauricio Cristian. (2017). Educación por habilidades: Perspectivas y retos para el sistema educativo. Revista Educación, 41(2), 1-22. https://doi.org/10.15517/revedu.v41i2.21719

Programa de Naciones Unidas para el Desarrollo. (2013). Informe Sobre Desarrollo Humano 2013. El Ascenso del Sur: Progreso Humano en un Mundo Diverso. https://bit.ly/2XGwbsj

Propp, Vladimir. (2015). Morfología del cuento. Ciudad de México: Colofón.

Roser, Max., and Esteban, Ortiz-Ospina. (2018). Our world in data. https://ourworldindata.org/literacy

Rubin, Beth Cara., and Giarelli, James M. (Eds.) (2015). Civic education for diverse citizens in global times: rethinking theory and practice. Lawrence Erlbaum Associates. https://doi.org/10.4324/9780203826911

Rychen, Dominique Simon., and Salganik, Laura Hersh. (2003). Key Competencies for a Successful Life and a Well-Functioning Society. Hogrefe Publishing. Recuperado de: https://bit.ly/318tmpi 
Sarica, Hatice Çirali., and Usluel, Yasemin Koçak. (2016). The effect of digital storytelling on visual memory and writing skills. Computers and Education, 94, 298-309. https://doi.org/10.1016/j.compedu.2015.11.016

Scalise, Kathleen. (2016). Student collaboration and school educational technology: Technology integration practices in the classroom. I-Manager's Journal on School Educational Technology, 11(4), 53-63. https://doi.org/10.26634/jsch.11.4.6012

Schleicher, Andreas. (2018). PISA 2018: Insights and Interpretations. https://bit.ly/3b6FHMI

Schulz, Wolfram. (2012). Educación para la ciudadanía y participación ciudadana. Una presentación del estudio ICCS 2009 y sus resultados. En Nicolás De Alba Fernández, Francisco F. García Pérez, y Antoni Santisteban Fernández (Eds.), Educar para la participación ciudadana en la enseñanza de las ciencias sociales, (Vol. I; pp. 47-62). Sevilla: Diada. https://bit.ly/2XJXLom

Short, Barbara J. (2012). 21st century skills development: Learning in digital communities: Technology and collaboration (Dissertation Doctoral). Universidad de Oregon, Oregon, United States. https://bit.ly/3AJrOP7

Sunayama, Wataru., Takahashi, Mayu., y Kawamoto, Kayo. (2017). Marco de evaluación y mantenimiento de la motivación de aprendizaje por funciones de historia y vida [Publicado en japonés]. Revista de la Sociedad japonesa de teoría difusa e informática inteligente, 29(3), 586-594. https://doi.org/10.3156/jsoft.29.3 586

Taizan, Yu. (2019). Competencias y enseñanza del idioma japonés desde la perspectiva de las habilidades de pensamiento transversal [Publicado en japonés]. Educación de idioma japonés, 83, 6-8. https://doi.org/10.20555/kokugoka.83.0 6

Takamiya, Masaki. (2015). ¿Por qué es necesaria la educación cívica? Cuatro razones por las que necesitamos la educación cívica [Publicado en japonés]. En Gen, Ito. (Ed.), Aprender pedagogía a través del trabajo. Nakashika. Disponible en https://bit.ly/36H7GiF

Taras, Maddalena. (2005). Assessment - Summative and Formative - Some Theoretical Reflections. British Journal of Educational Studies, 53(4), 466-478. https://doi.org/10.1111/j.1467-8527.2005.00307.x

Tomita, Shohei. (2002). Real vs. No real. La comprensión de los niños de los personajes de fantasía [Publicado en japonés]. Revista japonesa de psicología del desarrollo, 13(2), 122-135. https://doi.org/10.11201/jjdp.13.122

United Nations Development Programme. (2020). Human development report. Recuperado de http://hdr.undp.org/en

Watanabe, Masako. (2004). La estructura del consentimiento: Estilos de expresión del pensamiento en la educación primaria japonesa y estadounidense [Publicado en japonés]. Toyokan. Disponible en https://bit.ly/3jSUFJM 
Watanabe, Yayoi. (2011). ¿Qué es el "muro de los 10 años" de los niños? Psicología del desarrollo para superarlo [Publicado en japonés]. Kobunsha. https://bit.ly/3cfSuu2

Wilcoxon, Frank. (1945). Individual comparisons by ranking methods. Biometrics Bulletin, 1(6), 80-83. https://doi.org/10.2307/3001968

Wilson, Mark., Gochyyev, Perman., and Scalise, Kathleen. (2017). Modeling data from collaborative assessments: Learning in digital interactive social networks. Journal of Educational Measurement, 54(1), 85-102. https://doi.org/10.1111/jedm.12134

Wilson, Mark., Scalise, Kathleen., and Gochyyev, Perman. (2016). Assessment of learning in digital interactive social networks: A learning analytics approach. Online Learning (Newburyport, Mass.), 20(2). file:///C:/Users/Rebeca/AppData/Local/Temp/799-4202-1PB.pdf 
Revista indizada en
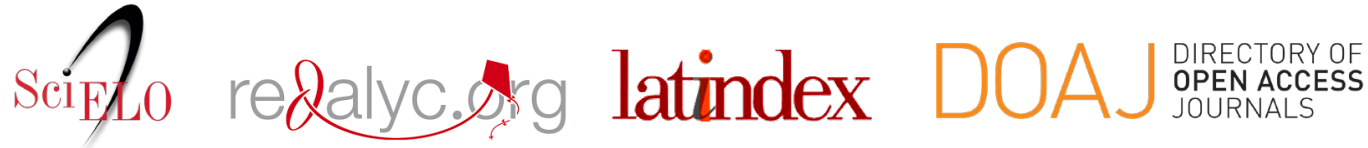

Distribuida en las bases de datos:
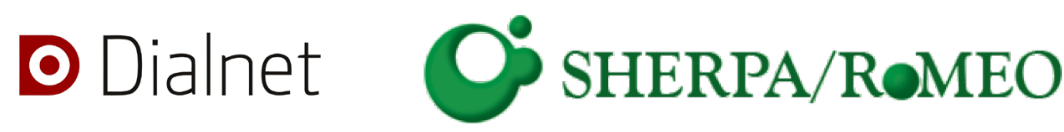

REDIB

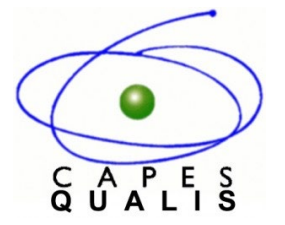

MIAR 